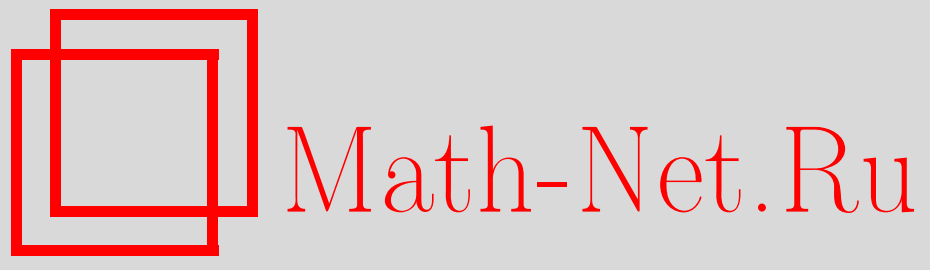

M. Ruiz-Medina, R. Crujeiras, A central limit result in the wavelet domain for minimum contrast estimation of fractal random fields, Теория вероятн. и ее примен., 2013, том 58, выпуск 3, 550-583

DOI: https://doi.org/10.4213/tvp4526

Использование Общероссийского математического портала Math-Net.Ru подразумевает, что вы прочитали и согласны с пользовательским соглашением http: //www . mathnet.ru/rus/agreement

Параметры загрузки:

IP: 3.95 .254 .165

26 апреля 2023 г., 12:31:31

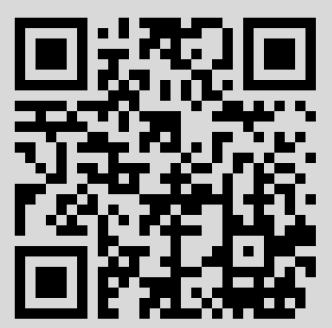


(C) 2013 г. RUIZ-MEDINA M. D.* , CRUJEIRAS R. M.**

\title{
A CENTRAL LIMIT RESULT IN THE WAVELET DOMAIN FOR MINIMUM CONTRAST ESTIMATION OF FRACTAL RANDOM FIELDS ${ }^{1)}$
}

\begin{abstract}
В статье [36] был получен критерий минимального контраста для состоятельных оценок пространственной фрактальной размерности для области вэйвлетов для класса полупараметрических фракталов гауссовых случайных полей, содержащих в качестве частных случаев фрактальное броуновское движение и процессы Рисса-Бесселя. В предлагаемой статье асимптотическая нормальность оценки минимального контраста получена с помощью центральной предельной теоремы для многомерных стохастических интегралов (см. [29]).
\end{abstract}

Ключевые слова и фразы: асимптотическая нормальность, оценка минимального контраста, область вейвлетов, винеровский xaoc.

1. Introduction and examples. The most well-known and widely used minimum contrast functional in the spectral domain is the Whittle or Gauss-Whittle discrepancy (see [39] for more examples of spectral contrast processes). For instance, [16] and [11] propose a minimum contrast estimator based on the Whittle approximation to the Gaussian log-likelihood for stationary random fields. This procedure has been extended to other contexts, involving long-range dependence, fractality or intermittency.

Whittle estimators for long-range dependent processes and fields have been studied by [22] and [20], respectively. In [20], the proposed estimator is obtained from a continuous version of the Whittle contrast function, supplied with a weight function and a similar idea has been also considered in [14], for Gaussian processes with stationary increments. In both cases, consistency and asymptotic normality of the estimators were derived. Similar results

${ }^{*}$ Department of Statistics and Operations Research, Faculty of Sciences, University of Granada, Spain; e-mail: mruiz@ugr.es

** Department of Statistics and Operations Research, Faculty of Mathematics, University of Santiago de Compostela, Spain; e-mail: rosa.crujeiras@usc.es

1) This work was supported by projects MTM2008-03010, MTM2009-13393, and MTM2012-32674 from the Spanish Ministry of Science, and by project P09-FQM-5052 of the Andalousian CICE, Spain. 
were also obtained by [5] for non-Gaussian processes with possible long-range dependence, based on second and third order cummulant spectra. For both long-range dependence and fractality, [4] studies a class of spectral-based minimum contrast estimators for fractional stationary stochastic processes and fields, such as the fractional Riesz-Bessel motion (fRBM) introduced in [3]. For quadratic forms of the bivariate Appell polynomials, and nonlinear transformations of stationary processes displaying long-range dependence, beyond the Gaussian case, Appell expansion provides a useful tool for deriving limit results (see, for instance, [15], [38] and [8]). Asymptotic properties of quasi-likelihood parameter estimators of stochastic processes, including the long-range dependent case, are also established in [18].

The references cited above present asymptotic results in an increasing domain framework, that is, assuming that the observation domain grows with the sample size. Alternatively, properties of the periodogram have been studied under fixed-domain asymptotics by [37]. In the same setting, [21] provides similar results for cross-periodograms of multivariate stationary random fields. In the parametric context, fixed-domain asymptotic properties and distribution of tapered maximum likelihood estimators for the covariance parameters have been studied by [12]. Note that results in [12] are restricted to stationary Gaussian processes observed on a bounded interval in $\mathbf{R}$, with special attention to the Ornstein-Uhlenbeck process. Fractal dimension estimation for stationary non-Gaussian random fields has been studied in [9], considering increment-based estimators.

Wavelet-based semiparametric estimation of the memory parameter, in the time series context, is addressed in [34], for the class of nonstationary $M(d)$ processes with memory parameter $d \in \mathbf{R}$. The $k$ th order difference of these processes is weakly stationary having spectral density factorized into a memory part and a short-range spectral density. Log-regression wavelet estimators and wavelet Whittle estimators are considered in the approximation of the memory parameter. Their asymptotic variance and asymptotic normality are derived. Fourier and wavelet approaches are compared in [13] for the estimation of the memory parameter $d$.

The wavelet-based minimum contrast estimation of a semiparametric class of Gaussian random fields, displaying local self-similar behavior as fractional Brownian motion, has been addressed in [36]. Weak consistency of the wavelet periodogram and the minimum contrast estimator are established for a family of Haar-type isotropic wavelet functions. An increasing domain asymptotic design is considered in the wavelet domain, with respect to the continuous translation parameter. This approach is equivalent to consider fixed domain asymptotics in the spatial domain.

In this work, the asymptotic normality of the class of wavelet-based minimum contrast estimators studied in [36] is derived. Specifically, central limit results for multiple stochastic integrals derived by [29] are applied to 
prove the asymptotic normality of the minimum contrast estimator. Note that the results in [29] were extended to the multidimensional case in [30] and [32], considering stochastic integrals with respect to scattered random measures. In addition, sufficient conditions for the convergence of sequences of multiple Itô-type integrals are established in [31] and [33]. A new characterization for sequences of vectors of multiple integrals is provided in [28], using Malliavin calculus. These developments constitute a powerful tool and have been recently applied to other contexts, such as the study of fractional stochastic integrals (see [10]) or Skorokhod integrals, as in [26].

The central limit result derived in this paper in the wavelet domain is based on the identification of the Wiener chaos of the semiparametric Gaussian random field class considered with the Wiener chaos of an isonormal process related to the wavelet random field. In the derivation of the asymptotic finite variance of the limit distribution, the properties of the wavelet family selected are crucial. The central limit results derived in [29] for multiple stochastic integrals are then applied to obtain the asymptotic normality of the proposed wavelet-based minimum contrast estimator. Note that, although the elements of the class of the semiparametric Gaussian random fields studied display local self-similarity, in the second-order moment sense, they are not necessarily stationary. Hence, the contrast estimation methodologies, based on the Fourier periodogram, usually developed in the spectral domain (see, for example, [20]) can not be applied in this setting. In particular, in the special case where the corresponding Reproducing Kernel Hilbert Space (RKHS) is isomorphic to a fractional Sobolev space, this fractal semiparametric class is included in the one introduced in [35].

This paper is organized as follows. In section 2, basic definitions and notation are introduced in relation to the wavelet Gaussian random field family considered. The wavelet-based minimum contrast estimation procedure is also described in this section. Section 3 is devoted to some technical conditions and preliminary results concerning the weak consistency of the wavelet periodogram and the minimum contrast parameter estimator. The identification of the wavelet random field with an isonormal process, and the corresponding Wiener chaos is obtained in section 4. Section 5 provides the main result on the asymptotic normality of the wavelet-based minimum contrast parameter estimator. Final remarks and future open research lines are discussed in section 6 .

2. Notation and background. In this section, general notation and background on random fields and on the wavelet scenario selected for the subsequent developments will be described (see also [36]).

2.1. Random fields. Let $H$ be a separable Hilbert space with inner product $\langle\cdot, \cdot\rangle_{H}$, and associated norm $\|\cdot\|_{H}$. Denote by $X$ the generalized random field (GRF) indexed by the elements of $H$, i.e., a centered Gaussian 
family defined on some probability space $(\Omega, \mathscr{F}, \mathbf{P})$, given by $\{X(\phi), \phi \in H\}$.

Since our interest is focused on statistical inference on the fractal parameter characterizing the local mean quadratic variation of $X, H$ is considered to be a fractional Sobolev space on a compact domain $D \subset \mathbf{R}^{d}$, namely $\bar{H}^{-\alpha}(D)$, for certain positive $\alpha$. Here,

$$
\bar{H}^{-\alpha}(D)=\left\{g \in H^{-\alpha}\left(\mathbf{R}^{d}\right): \operatorname{supp} g \subseteq D\right\},
$$

where supp $g$ denotes the support of function $g$, and $H^{-\alpha}\left(\mathbf{R}^{d}\right)$ is the fractional Sobolev space of order $(-\alpha)$ on $\mathbf{R}^{d}$. This space is constituted by the tempered distributions $g$ such that

$$
\left(1+\|\lambda\|^{2}\right)^{-\alpha / 2} \widehat{g}(\lambda) \in L^{2}\left(\mathbf{R}^{d}\right), \quad \lambda \in \mathbf{R}^{d},
$$

with $\widehat{g}$ being the weak-sense Fourier transform of $g$ defined by duality, and $L^{2}\left(\mathbf{R}^{d}\right)$ denoting the space of square-integrable functions on $\mathbf{R}^{d}$. The dual Hilbert space $H^{*}$ of $H$ is then given by $H^{\alpha}(D)$, the space of distributions $f$ on $D$ such that there exists a function $F \in H^{\alpha}\left(\mathbf{R}^{d}\right)$, whose restriction to domain $D$ coincides with $f$ (see, for example, [40], for more details). The isomorphic relationship of the RKHS of $X$, denoted by $\mathscr{H}(X)$, with the fractional Sobolev space $H^{\alpha}(D)=\left[\bar{H}^{-\alpha}(D)\right]^{*}$ implies that there exist positive constants $0<C_{1} \leqslant C_{2}$ such that

$$
C_{1}\|f\|_{\bar{H}^{-\alpha}(D)} \leqslant\|f\|_{[\mathscr{H}(X)]^{*}}=B_{X}(f, f) \leqslant C_{2}\|f\|_{\bar{H}^{-\alpha}(D)} \quad \forall f \in \bar{H}^{-\alpha}(D) .
$$

In particular, for $\alpha>d / 2$, from embedding theorems between fractional Besov spaces (c.f., [40]), the functions in the RKHS of $X$ are Hölder continuous of order $(\alpha-d / 2)$. Therefore, the Dirac delta distribution belongs to $\bar{H}^{-\alpha}(D)$, which defines the support of the covariance operator $R_{X}$ of $X$, given by,

$$
R_{X}(\phi)(\psi)=B_{X}(\phi, \psi) \quad \forall \phi, \psi \in \bar{H}^{-\alpha}(D) .
$$

Thus, we can univocally define a zero-mean ordinary random field, $\left\{\widetilde{X}(x), x \in D \subseteq \mathbf{R}^{d}\right\}$, in terms of the following identity:

$$
\widetilde{X}(x)=X\left(\delta_{x}\right)=\int_{D} \tilde{X}(y) \delta_{x}(y) d y \quad \forall x \in D,
$$

where the covariance kernel $B_{\tilde{X}}^{\alpha}$ of $\tilde{X}$ is determined from

$$
B_{\tilde{X}}^{\alpha}(x, y)=B_{X}\left(\delta_{x}, \delta_{y}\right)=\int_{D} \int_{D} B_{\tilde{X}}^{\alpha}(z, v) \delta_{x}(z) \delta_{y}(v) d z d v \quad \forall x, y \in D .
$$

Here, $\delta_{u}(\cdot)$ denotes the Dirac delta distribution at point $u \in D$. The meansquare continuity of random field $\widetilde{X}$ follows from the continuity of $B_{\tilde{X}}^{\alpha}$, since, as commented, from equation (2.1), $\alpha>d / 2$, the functions in $\mathscr{H}(X)$ are 
Hölder continuous of order $(\alpha-d / 2)$, and, by definition of $B_{\tilde{X}}^{\alpha}$ in equation $(2.2), B_{\tilde{X}}^{\alpha} \in \mathscr{H}(X) \otimes \mathscr{H}(X)$. Therefore, equation (2.2) univocally determines the ordinary random field $\widetilde{X}$, due to its continuity. In particular, $X$ admits the integral representation

$$
X(\phi)=\int_{D} \tilde{X}(x) \phi(x) d x \quad \forall \phi \in \bar{H}^{-\alpha}(D),
$$

as well as its covariance function $B_{X}$

$$
B_{X}(\phi, \psi)=\int_{D} \int_{D} B_{\tilde{X}}(x, y) \phi(y) \psi(x) d x d y \quad \forall \phi, \psi \in \bar{H}^{-\alpha}(D),
$$

in terms of $B_{\tilde{X}}$ satisfying $B_{\tilde{X}}(x, y)=\mathbf{E}[\tilde{X}(x) \widetilde{X}(y)]$, for $x, y \in D$. In the stationary case, for $\widetilde{X}$ defined on $\mathbf{R}^{d}$, and $\alpha \in(d / 2, d)$, the inequality in (2.1) means that

$$
C_{1}\left(1+|\lambda|^{2}\right)^{-\alpha} \leqslant f_{\tilde{X}}(\lambda) \leqslant C_{2}\left(1+|\lambda|^{2}\right)^{-\alpha},
$$

where $f_{\tilde{X}}=\mathscr{F}\left(B_{\tilde{X}}\right)$ denotes the Fourier transform of $B_{\tilde{X}}$ (i.e., the spectral density of $\widetilde{X})$.

The estimation problem considered here is associated with a semiparametric family of centered Gaussian random fields with covariance function $\left\{B_{\tilde{X}}^{\alpha}(\cdot, \cdot)=B_{\tilde{X}}(\alpha, \cdot, \cdot), \alpha \in(d / 2, d)\right\}$, satisfying

$$
\frac{B_{\tilde{X}}^{\alpha}(x, y)}{\|x-y\|^{2 \alpha-d}} \longrightarrow C \quad \text { as }\|x-y\| \longrightarrow 0,
$$

for a certain positive constant $C$. Specifically, the asymptotic normality of the wavelet-based minimum contrast estimator of $\alpha$, proposed in [36], is derived. The estimation results formulated in [36] allow the consistent approximation of the fractal parameter $(\alpha-d / 2)$ characterizing the local regularity of the sample-paths (see [1]), as well as of the functions in the associated RKHS. In particular, when an isomorphic identification between the RKHS and the fractional Sobolev space $H^{\alpha}(D)$ of order $\alpha$ can be established, we obtain the minimum contrast estimation of the parameter $\alpha$ characterizing the class of generalized random fields introduced in [35].

2.2. Wavelet scenario. The minimum contrast estimation procedure for $\alpha$ is formulated in the wavelet domain, based on the wavelet periodogram, and some considerations concerning the wavelet basis selected must be introduced. The Continuous Discrete Wavelet Transform (CDWT) is used in our development. This transform is defined by discretizing the scale of the continuous wavelet transform (CWT) on a dyadic grid (see [24] and [25], among others). Hölder regularity can be analyzed in terms of this discretescale based transform (see [2], [6], [23], among others). Here, compactly 
supported wavelets are considered in the definition of the CDWT. For simplicity and without loss of generality, assume that the compact domain $D$, where the wavelet functions are defined, is of the form $D=[-M, M]^{d}$, for a certain positive constant $M$, being then $(2 M)^{d}$ its Lebesgue measure. In the isotropic case, we consider the CDWT defined in terms of a basic wavelet function $\boldsymbol{\Psi}$, which generates by multidimensional translations and one-dimensional dyadic dilations or contractions a wavelet basis of $L^{2}(D)$. Specifically, for each resolution level $j$, and for every $x \in D$, let us denote by

$$
\boldsymbol{\Psi}_{j, b}(x)=\boldsymbol{\Psi}_{j, b}(\|x\|)=2^{j d / 2} \boldsymbol{\Psi}\left(\left\|2^{j} x-b\right\|\right), \quad b \in L_{j}, \quad j \in \mathbf{Z},
$$

a wavelet function on $\mathbf{R}^{d}$, translated at the center $b$, for the resolution level $j$, where $L_{j}$ is the domain of $\mathbf{R}^{d}$ containing the continuous translation parameter $b$. Indeed, since the support $D$ of the basic wavelet functions is assumed to be a $d$-dimensional interval, the domain $L_{j}$ can be defined as $L_{j}=\left[0,2^{j}\right]^{d}$. Hence, as $j \rightarrow \infty$, the set $L_{j}$ becomes the $d$-dimensional space $\mathbf{R}_{+}^{d}$.

Note that, in the anisotropic case, orthonormal wavelet bases on $\mathbf{R}^{d}$ can be generated from the tensorial product of one-dimensional wavelet functions. In general, in the anisotropic case, one basic wavelet function is considered for each one of the main directions.

The asymptotic results derived in this paper hold for an increasing resolution level, $j \rightarrow \infty$, i.e., the increasing domain asymptotic framework is adopted in the wavelet domain. This approach corresponds to a fixed domain asymptotic setting in the spatial domain $D$.

For each resolution level $j \in \mathbf{Z}$, associated with the dyadic scale $2^{-j}$, consider the CDWT of $\tilde{X}$ in equation (2.3) given by

$$
\begin{aligned}
X\left(\boldsymbol{\Psi}_{j, b}\right) & =\int_{D} \tilde{X}(x) \boldsymbol{\Psi}_{j, b}(x) d x \\
& =\int_{D} 2^{j d / 2} \tilde{X}(x) \boldsymbol{\Psi}\left(2^{j} x-b\right) d x, \quad b \in L_{j} \subset \mathbf{R}_{+}^{d} .
\end{aligned}
$$

Consider also the semiparametric covariance family defined in equation (2.5), and denote by $\alpha_{0}$ the true parameter value. The associated twodimensional CDWT at scale $2^{-j}$ is defined as

$$
\begin{aligned}
B_{X}^{\alpha_{0}}\left(\boldsymbol{\Psi}_{j, b}, \boldsymbol{\Psi}_{j, u}\right) & =\mathbf{E}\left[X\left(\boldsymbol{\Psi}_{j, b}\right) X\left(\boldsymbol{\Psi}_{j, u}\right)\right] \\
& =\int_{D \times D} B_{\tilde{X}}^{\alpha_{0}}(x, y) \boldsymbol{\Psi}_{j, b}(x) \boldsymbol{\Psi}_{j, u}(y) d x d y \\
& =\int_{D \times D} B_{\tilde{X}}^{\alpha_{0}}(x, y) 2^{j d / 2} \mathbf{\Psi}\left(2^{j} x-b\right) 2^{j d / 2} \mathbf{\Psi}\left(2^{j} y-u\right) d x d y
\end{aligned}
$$

where $X\left(\boldsymbol{\Psi}_{j, c}\right)$, for $c=b, b \in L_{j}$, is given as in equation (2.6), for each $j \in \mathbf{Z}$.

The wavelet periodogram provides a nonparametric estimator of the diagonal of the two-dimensional $(2 d-)$ wavelet transform of the covariance 
function (see [24]). Specifically, in our setting, the wavelet periodogram at resolution level $j \in \mathbf{Z}$ and location $b \in L_{j}$ is defined as

$$
S(j, b, b)=\left|\int_{D} \tilde{X}(x) \boldsymbol{\Psi}_{j, b}(x) d x\right|^{2}=\left|\int_{D} 2^{j d / 2} \tilde{X}(x) \mathbf{\Psi}\left(2^{j} x-b\right) d x\right|^{2} .
$$

The expected value of the wavelet periodogram (2.7) is then computed as

$$
\left.\mathbf{E}(S(j, b, b))=\left.\mathbf{E}\left[\mid X\left(\boldsymbol{\Psi}_{j, b}\right)\right)\right|^{2}\right]=B_{X}^{\alpha_{0}}\left(\boldsymbol{\Psi}_{j, b}, \mathbf{\Psi}_{j, b}\right), \quad b \in L_{j}, \quad j \in \mathbf{Z} .
$$

The compactly supported wavelet basis selected must be such that the above integrals, defining the two-dimensional CDWT of the covariance function, are finite. It is also assumed that a continuous and discrete version of the associated wavelet transform are available with the same values on the nodes of the corresponding regular dyadic grid associated with the discrete version.

3. Preliminary results and technical conditions. The conditions assumed and the consistency results derived in [36], as well as the conditions needed for the asymptotic normality of the minimum contrast estimator (3.11) are now introduced.

3.1. Conditions. The following assumptions are made on the Gaussian random field family and wavelet transform considered in the subsequent developments:

A.1. The covariance function of the centered Gaussian random field $\tilde{X}$ introduced in equation (2.3) satisfies (2.5).

A.2. The wavelet basis selected is an isotropic wavelet basis with the basic wavelet function $\boldsymbol{\Psi}$ satisfying

$$
\boldsymbol{\Psi}(\|z\|)=\left\{\begin{aligned}
1, & 0 \leqslant\|z\|<1 / 2, \\
-1, & 1 / 2 \leqslant\|z\|<1, \\
0 & \text { otherwise. }
\end{aligned}\right.
$$

The results below hold for any isotropic wavelet basis with function $\Psi$ displaying a similar behavior to the one-dimensional Haar mother wavelet, with respect to the modulus function. That is, $\boldsymbol{\Psi}$ as a function of the modulus behaves like a linear combination of weighted indicator functions of the elements of a partition of a closed interval.

Assumptions A.1 and A.2 allow the explicit computation of the asymptotic orders, when $j \rightarrow \infty$, of the CDWT of the semiparametric covariance function family (2.5) (see [36] and Lemma 3.1 below).

For deriving the consistency of the minimum contrast estimator introduced in (3.11), the following additional conditions are considered in Proposition 2 in $[36]$ : 
A.3. The weight function $\omega \in L^{1}\left(\mathbf{R}_{+}^{d}\right)$ is such that

$$
j \int_{L_{j}}|w(b)| d b \longrightarrow C, \quad j \rightarrow \infty, \quad C \geqslant 0 .
$$

Note that a sufficient condition for A.3 to hold is, for example,

$$
\int_{L_{j}}|w(b)| d b=\mathscr{O}\left(j^{-\beta}\right), \quad \beta \geqslant 1 .
$$

A.4. There exists a sequence of functions $\left\{v_{j}\right\}$ on $L_{j}$ such that the sequence

$$
\left\{h^{j}(\alpha, b)=\frac{v_{j}(b)}{B_{X}^{\alpha}\left(\mathbf{\Psi}_{j, b}, \mathbf{\Psi}_{j, b}\right)}\right\}
$$

is equicontinuous with respect to the first argument, i.e.,

$$
\left|h^{j}\left(\alpha_{1}, b\right)-h^{j}\left(\alpha_{2}, b\right)\right| \leqslant \eta\left(\left|\alpha_{1}-\alpha_{2}\right|\right)
$$

being $\eta$ the modulus of continuity, independently of the argument $b$. In addition, the integral

$$
\int_{L_{j}}|S(j, b, b)|\left|\frac{\omega(b)}{v_{I}(b)}\right| d b=C_{j} \underset{P_{\alpha_{0}}}{\longrightarrow} C_{\infty}<\infty, \quad j \rightarrow \infty,
$$

where $\underset{P_{\alpha_{0}}}{\longrightarrow}$ denotes convergence in probability, and $v_{I}$ is the inferior envelope of $\left\{v_{j}\right\}$, that is, $v_{I}=\inf _{j \in \mathbf{Z}} v_{j}$.

It should be noted that no identifiability conditions are here assumed, due to the design of the contrast function $\mathscr{V}$ (see equation (3.9) below), which is positive and has a unique minimum under assumptions A.1 and A.2, in view of Lemma 3.1.

Finally, for the asymptotic normality of the minimum contrast estimator, the following additional conditions are required.

B.1. Function $B_{X}^{\alpha}\left(\boldsymbol{\Psi}_{j, b}, \mathbf{\Psi}_{j, b}\right), \alpha \in \Lambda$, is twice differentiable with respect to $\alpha$, for any $b \in L_{j}$, and $j \geqslant j_{0}$, for a certain $j_{0} \in \mathbf{Z}$.

B.2. The weight function $\omega \in L^{1}\left(\mathbf{R}_{+}^{d}\right) \cap C\left(\mathbf{R}_{+}^{d}\right)$ is such that, for $l, m=$ $1, \ldots, 4$,

$$
I_{l, m}(j)=\int_{\mathscr{S}_{l, m}(j)} \omega\left(b_{1}\right) \omega\left(b_{1}-y\right) \omega\left(b_{1}-h\right) \omega\left(b_{1}-(h-x)\right) d b_{1} d h d x d y \longrightarrow 0
$$

as $j \rightarrow \infty$, where $\mathscr{S}_{l, m}(j)=D_{l, m}(j) \times D_{h}(x, y, j) \times D_{b_{1}}(h-x, y, h, j)$, with

$$
\begin{aligned}
D_{h}(x, y, j) & =\left\{h \in \prod_{i=1}^{d}\left[x_{i}-2^{j}, x_{i}+2^{j}\right] \cap \prod_{i=1}^{d}\left[y_{i}-2^{j}, y_{i}+2^{j}\right]\right\} \\
& =\prod_{i=1}^{d}\left[\left(x_{i} \vee y_{i}\right)-2^{j},\left(x_{i} \wedge y_{i}\right)+2^{j}\right],
\end{aligned}
$$




$$
\begin{aligned}
& D_{b_{1}}(h-x, y, h, j)=\left\{b_{1} \in \prod_{i=1}^{d}\left[h_{i}-x_{i}, h_{i}-x_{i}+2^{j}\right] \cap \prod_{i=1}^{d}\left[y_{i}, y_{i}+2^{j}\right]\right. \\
& \left.\cap \prod_{i=1}^{d}\left[h_{i}, h_{i}+2^{j}\right]\right\} \\
& =\prod_{i=1}^{d}\left[h_{i}-x_{i} \vee y_{i} \vee h_{i},\left(h_{i}-x_{i} \wedge y_{i} \wedge h_{i}\right)+2^{j}\right] \\
& D_{1,1}(j)=\left[-2^{j+1}-\frac{R}{\sqrt{d}}, 2^{j+1}+\frac{R}{\sqrt{d}}\right]^{d}\left(B_{R}(0)\right)^{-1} \\
& \times\left[-2^{j+1}-\frac{R}{\sqrt{d}}, 2^{j+1}+\frac{R}{\sqrt{d}}\right]^{d}\left(B_{R}(0)\right)^{-1}, \\
& D_{1,2}(j)=\left[-2^{j+1}-\frac{R}{\sqrt{d}}, 2^{j+1}+\frac{R}{\sqrt{d}}\right]^{d}\left(B_{R}(0)\right)^{-1} \times B_{R}(0), \\
& D_{1,3}(j)=B_{R}(0) \times\left[-2^{j+1}-\frac{R}{\sqrt{d}}, 2^{j+1}+\frac{R}{\sqrt{d}}\right]^{d}\left(B_{R}(0)\right)^{-1}, \\
& D_{1,4}(j)=D_{1,4}=B_{R}(0) \times B_{R}(0), \\
& D_{2,1}(j)=D_{y}(j, R, d)\left(B_{R}(0)\right)^{-1} \times\left[-2^{j+1}-\frac{R}{\sqrt{d}}, 2^{j+1}+\frac{R}{\sqrt{d}}\right]^{d}\left(B_{R}(0)\right)^{-1} \text {, } \\
& D_{2,2}(j)=D_{y}(j, R, d)\left(B_{R}(0)\right)^{-1} \times B_{R}(0), \\
& D_{2,3}(j)=B_{R}(0) \times\left[-2^{j+1}-\frac{R}{\sqrt{d}}, 2^{j+1}+\frac{R}{\sqrt{d}}\right]^{d}\left(B_{R}(0)\right)^{-1} \text {, } \\
& D_{2,4}(j)=D_{2,4}=B_{R}(0) \times B_{R}(0), \\
& D_{3,1}(j)=\left[-2^{j+1}-\frac{R}{\sqrt{d}}, 2^{j+1}+\frac{R}{\sqrt{d}}\right]^{d}\left(B_{R}(0)\right)^{-1} \times D_{x}(j, R, d)\left(B_{R}(0)\right)^{-1}, \\
& D_{3,2}(j)=\left[-2^{j+1}-\frac{R}{\sqrt{d}}, 2^{j+1}+\frac{R}{\sqrt{d}}\right]^{d}\left(B_{R}(0)\right)^{-1} \times B_{R}(0), \\
& D_{3,3}(j)=B_{R}(0) \times D_{x}(j, R, d)\left(B_{R}(0)\right)^{-1}, \\
& D_{3,4}(j)=D_{3,4}=B_{R}(0) \times B_{R}(0) \text {, } \\
& D_{4,1}(j)=D_{y}(j, R, d)\left(B_{R}(0)\right)^{-1} \times D_{x}(j, R, d)\left(B_{R}(0)\right)^{-1} \text {, } \\
& D_{4,2}(j)=D_{y}(j, R, d)\left(B_{R}(0)\right)^{-1} \times B_{R}(0), \\
& D_{4,3}(j)=B_{R}(0) \times D_{x}(j, R, d)\left(B_{R}(0)\right)^{-1} \text {, } \\
& D_{4,4}(j)=D_{4,4}=B_{R}(0) \times B_{R}(0) \text {, } \\
& D_{z}(j, R, d)=\left\{z \in\left[-2^{j+1}-2^{j}, 2^{j+1}-\frac{R}{\sqrt{d}}\right]^{d} \cap\left[-2^{j+1}+\frac{R}{\sqrt{d}}, 2^{j+1}+2^{j}\right]^{d}\right\} \\
& =\left[\left(-2^{j+1}-2^{j}\right) \vee\left(-2^{j+1}+\frac{R}{\sqrt{d}}\right),\left(2^{j+1}-\frac{R}{\sqrt{d}}\right) \wedge\left(2^{j+1}+2^{j}\right)\right]^{d} .
\end{aligned}
$$


$\mathrm{R}$ e $\mathrm{m}$ a r k 3.1. Note that, under assumption A3, condition B2 holds, for example, if the weight function $\omega \in L^{1}\left(\mathbf{R}_{+}^{d}\right) \cap C\left(\mathbf{R}_{+}^{d}\right)$ is symmetric with respect to zero.

3.2. Some preliminary results. We include in this section a lemma providing the asymptotic properties, when the resolution level increases, of the two-dimensional CDWT of the covariance (2.5), and two propositions on the consistency of the wavelet periodogram and the minimum contrast estimator. The proofs of these results can be found in [36].

Denote by $W_{\alpha_{0}-d / 2}$ the fractional Brownian motion with Hurst parameter $\left(\alpha_{0}-d / 2\right)$. Under condition $A .1, W_{\alpha_{0}-d / 2}$ displays the same local behavior as the ordinary random field $\tilde{X}$ in $(2.3)$.

Lemma 3.1 (see [36]). (i) Under condition A.1, as $j \rightarrow \infty$, the following asymptotic approximation holds:

$$
B_{X}^{\alpha_{0}}\left(\boldsymbol{\Psi}_{j, b}, \mathbf{\Psi}_{j, u}\right) \simeq\left[2^{-j}\right]^{2 \alpha_{0}} C_{\boldsymbol{\Psi}}\left(\alpha_{0}, b, u, j\right), \quad b \in L_{j},
$$

where

$$
\begin{aligned}
\lim _{j \rightarrow \infty} C_{\boldsymbol{\Psi}}\left(\alpha_{0}, b, u, j\right) & =C_{\boldsymbol{\Psi}}\left(\alpha_{0}, b-u, \infty\right)=\int_{\mathbf{R}^{d}}\|h\|^{2 \alpha_{0}-d} \gamma_{\mathbf{\Psi}}(h-(b-u)) d h \\
& =\left\langle\boldsymbol{\Psi}_{0, b}, \mathbf{\Psi}_{0, u}\right\rangle_{\left[\mathscr{H}_{W_{\alpha_{0}-d / 2}}\right]^{*}}
\end{aligned}
$$

with

$$
\gamma_{\mathbf{\Psi}}(h-(b-u))=\int_{\mathbf{R}^{d}} \boldsymbol{\Psi}(z) \boldsymbol{\Psi}(z-h+(b-u)) d z,
$$

and $\mathscr{H}_{W_{\alpha_{0}-d / 2}}$ denoting the RKHS of the fractional Brownian motion $W_{\alpha_{0}-d / 2}$.

(ii) Moreover, under condition A.2, for $\|b-u\|>>1$,

$$
\begin{aligned}
C_{\boldsymbol{\Psi}}\left(\alpha_{0}, b, u, \infty\right) & =\int_{\mathbf{R}^{d}}\|z\|^{2 \alpha_{0}-d}\left[\int_{\mathbf{R}^{d}} \boldsymbol{\Psi}(x) \boldsymbol{\Psi}(x-z+(b-u)) d x\right] d z \\
& \sim \mathscr{O}\left(\|b-u\|^{2 \alpha_{0}-3 d}\right) .
\end{aligned}
$$

Otherwise,

$$
C_{\Psi}\left(\alpha_{0}, b, u, \infty\right)=\frac{1}{2 \alpha_{0}\left(2 \alpha_{0}+d\right)} \xi_{v}(b-u)
$$

with

$$
\begin{aligned}
\xi_{v}(b-u)= & \|b-u-v\|^{2 \alpha_{0}+d}-4(\|b-u-v / 2\|)^{2 \alpha_{0}+d} \\
& +6\|b-u\|^{2 \alpha_{0}+d}-4\|b-u+v / 2\|^{2 \alpha_{0}+d} \\
& +\|b-u+v\|^{2 \alpha_{0}+d}
\end{aligned}
$$

for $v \in \mathbf{R}_{+}^{d}$ such that $\|v\|=1$. In particular,

$$
\lim _{j \rightarrow \infty} C_{\boldsymbol{\Psi}}\left(\alpha_{0}, b, j\right)=C_{\boldsymbol{\Psi}}\left(\alpha_{0}, \infty\right)=\widetilde{L}_{d}\left(\alpha_{0}\right)=\frac{1-2^{-\left(2 \alpha_{0}+d-2\right)}}{\alpha_{0}\left(2 \alpha_{0}+d\right)}
$$


Lemma 3.1 will also be applied in the proof of the main result of this paper, Theorem 5.1.

Lemma 3.2 (see [36]). Assume that conditions A.1 and A.2 hold. Let $\omega$ be a weight function such that the integral in (3.8) below is well defined. Then, as $j \rightarrow \infty$, for $\omega \in L^{1}\left(\mathbf{R}_{+}^{d}\right)$, the following limit holds in probability:

$$
\int_{L_{j}} \omega(b)\left[S(j, b, b)-B_{X}^{\alpha_{0}}\left(\boldsymbol{\Psi}_{j, b}, \boldsymbol{\Psi}_{j, b}\right)\right] d b \longrightarrow 0 .
$$

The proof is derived in [36] from the Hermite expansion of the wavelet periodogram applying Lemma 3.1.

3.3. Minimum contrast estimation. For the class of random fields considered, and in the wavelet scenario previously established, the authors in [36] propose a wavelet-based minimum contrast estimator of $\alpha_{0}$. Following the results in [17], they define the contrast function:

$$
\mathscr{V}\left(\alpha, \alpha_{0}\right)=-\int_{\mathbf{R}^{d}}\left[\ln \left(\frac{\widetilde{L}_{d}\left(\alpha_{0}\right)}{\widetilde{L}_{d}(\alpha)}\right)-\frac{\widetilde{L}_{d}\left(\alpha_{0}\right)}{\widetilde{L}_{d}(\alpha)}+1\right] \omega(b) d b,
$$

where $\omega$ is a weight function assumed to be in $L^{1}\left(\mathbf{R}_{+}^{d}\right)$. It can be seen that from definition of function $\widetilde{L}_{d}$ on $(d / 2, d)$ as in equation (3.7), the contrast function $\mathscr{V}$ in (3.9) is positive and has a unique minimum in $\alpha=\alpha_{0}$.

The sequence of random variables $\left\{U_{j}(\alpha), j \in \mathbf{Z}\right\}$ given, for all $\alpha \in$ $(d / 2, d)$, by

$$
U_{j}(\alpha)=\int_{L_{j}}\left[\ln \left(B_{X}^{\alpha}\left(\boldsymbol{\Psi}_{j, b}, \boldsymbol{\Psi}_{j, b}\right)\right)+\frac{S(j, b, b)}{B_{X}^{\alpha}\left(\mathbf{\Psi}_{j, b}, \mathbf{\Psi}_{j, b}\right)}\right] \omega(b) d b, \quad j \in \mathbf{Z}_{+},
$$

defines a contrast process for the contrast function $\mathscr{V}$, since

$$
\begin{gathered}
U_{j}(\alpha)-U_{j}\left(\alpha_{0}\right)=-\int_{L_{j}}\left(\ln \frac{B_{X}^{\alpha_{0}}\left(\boldsymbol{\Psi}_{j, b}, \boldsymbol{\Psi}_{j, b}\right)}{B_{X}^{\alpha}\left(\mathbf{\Psi}_{j, b}, \mathbf{\Psi}_{j, b}\right)}-\frac{S(j, b, b)}{B_{X}^{\alpha}\left(\boldsymbol{\Psi}_{j, b}, \mathbf{\Psi}_{j, b}\right)}\right. \\
\left.+\frac{S(j, b, b)}{B_{X}^{\alpha_{0}}\left(\boldsymbol{\Psi}_{j, b}, \boldsymbol{\Psi}_{j, b}\right)}\right) \omega(b) d b
\end{gathered}
$$

converges in probability to the contrast function, as $j \rightarrow \infty$ (see [36]). The minimum contrast estimator $\widetilde{\alpha}_{j}$ is then formulated, for each resolution level $j$, as

$$
\widetilde{\alpha}_{j}=\arg \min _{\alpha \in(d / 2, d)} U_{j}(\alpha) .
$$

The weak-consistency of the Fourier periodogram has been obtained in [14] for nonstationary Gaussian processes with stationary increments. Lemma 1 in [4] also provides parallel results in the Fourier spectral domain from continuous observations (for both random processes and fields). In addition, Proposition 1 in [20] gives an analogous result, in terms of the Fourier 
periodogram, for strictly stationary random fields. Lemma 3.2 establishes a consistency result for the wavelet periodogram. In all the cited references, the weak-consistency of the periodogram leads to the weak-consistency of the minimum contrast estimator proposed, under suitable conditions on the weight function family. For instance, in the spectral domain, [14] obtain the weak consistency of a minimum contrast estimator for a process with stationary increments. Analogous results were derived by [4] and [20] for Gaussian processes and fields. Finally, we refer to Lemma 3.3 below, obtained in [36], on the weak consistency of the wavelet-based minimum contrast estimator (3.11).

Lemma 3.3 (see [36]). Under conditions A.1-A.4, as $j \rightarrow \infty$,

$$
\widetilde{\alpha}_{j} \longrightarrow \alpha_{0}, \quad \text { in probability }
$$

4. Generalized Gaussian random fields and isonormal processes. The methodological lines established in [19] are followed in the derivation of the main result of this section, Lemma 4.3. This lemma provides the identification of the wavelet Gaussian random field family previously introduced with an isonormal process family. The associated Wiener chaos are also connected. For a detailed review on Wiener chaos and stochastic integrals, see [27].

Let us first consider the following definitions.

$\mathrm{D}$ e f i $\mathrm{n}$ i t i o $\mathrm{n}$ 4.1. Let $H$ be a separable Hilbert space. The set of random variables $\{\eta(h): h \in H\}$ is said to be an isonormal process on $H$ if $\eta$ is a centered $H$-indexed Gaussian family defined on a probability space $(\Omega, \mathscr{F}, \mathbf{P})$, and it satisfies

$$
\mathbf{E}[\eta(h) \eta(g)]=\langle h, g\rangle_{H},
$$

for every $h, g \in H$.

For $n \geqslant 1$, let $H^{\otimes n}$ be the $n$th tensor product of $H$, and let $H^{\odot n}$ be the $n$th symmetric tensor product of $H$. Denote by $H_{n}$ the $n$th Hermite polynomial.

$\mathrm{D}$ e f i $\mathrm{n}$ i t i o $\mathrm{n}$ 4.2. For an isonormal process $\eta$, the $n$th Wiener chaos $\mathscr{H}_{n}$ is defined as the closed linear subspace of $L^{2}(\Omega, \mathscr{F}, \mathbf{P})$ generated by the random variables $\left\{H_{n}(\eta(h)), h \in H,\|h\|_{H}=1\right\}$. The space $L^{2}(\Omega, \mathscr{G}, \mathbf{P})$ can then be decomposed into the infinite orthogonal sum of the subspaces $\mathscr{H}_{n}$ :

$$
L^{2}(\Omega, \mathscr{G}, P)=\bigoplus_{n=0}^{\infty} \mathscr{H}_{n}
$$

where $\mathscr{G}$ denotes the $\sigma$-field generated by the random variables $\{\eta(h), h \in H\}$. 
The multiple Wiener-Itô stochastic integral of order $n$ with respect to an isonormal process $\eta$, denoted by $I_{n}^{\eta}$, provides an isometry between the $n$th Wiener chaos associated to $\eta$ and $H^{\odot n}$. For every $n \geqslant 1$, the norm considered in $H^{\odot n}$ is given by $\sqrt{n !}\|\cdot\|_{H^{\otimes n}}$. The following result in [29] will be used for obtaining the asymptotic distribution of the minimum contrast estimator.

Lemma 4.1 (see [29, Theorem 1]). Assume that $\eta$ is an isonormal process on a separable Hilbert space $H$. Denote by $I_{n}^{\eta}(f)$ the multiple WienerItô stochastic integral (of order $n$ ) of $f$ with respect to $\eta$, and fix $n \geqslant 2$. For any sequence of elements $\left\{f_{m}, m \geqslant 1\right\}$ such that $f_{m} \in H^{\odot n}$, for every $m \geqslant 1$, and

$$
\lim _{m \rightarrow \infty} n !\left\|f_{m}\right\|_{H^{\otimes n}}^{2}=\lim _{m \rightarrow \infty} \mathbf{E}\left[I_{n}^{\eta}\left(f_{m}\right)^{2}\right]=\sigma_{n}^{2}
$$

the following conditions are equivalent:

(i) $\lim _{m \rightarrow \infty} \mathbf{E}\left[I_{n}^{\eta}\left(f_{m}\right)^{4}\right]=3\left[\sigma_{n}^{2}\right]^{2}$;

(ii) $\lim _{m \rightarrow \infty}\left\|f_{m} \otimes_{p} f_{m}\right\|_{H \otimes 2(n-p)}^{2}=0$, for $p=1, \ldots, n-1$, with $H^{\otimes 2(n-p)}$ denoting, as before, the $2(n-p)$ th tensor product of $H$, and $f_{m} \otimes_{p} f_{m}$ being the pth self-contraction of $f_{m}$;

(iii) the sequence $\left\{I_{n}^{\eta}\left(f_{m}\right), m \geqslant 1\right\}$ converges, as $m \rightarrow \infty$, to a centered Gaussian random variable with variance $\sigma_{n}^{2}$.

In order to derive the main result in this paper (Theorem 5.1), some statements on the identification of Gaussian generalized random fields with isonormal processes are needed. These results, as well as some other statements for their respective Wiener chaos are now introduced (see [41, Lemmas 1.1 and 1.2]) and [19].

Theorem 4.1. Let $\xi: \Omega \rightarrow \mathscr{B}$ be a centered Gaussian random element, which maps a probability space $(\Omega, \mathscr{F}, \mathbf{P})$ into a real separable Banach space $\mathscr{B}$, and $\operatorname{supp} \xi=\mathscr{B}$. Then, there exists a separable Hilbert space $H$ and an isonormal process $\{\eta(h): h \in H\}$ such that

$$
\left\langle\xi, x^{*}\right\rangle \in\{\eta(h): h \in H\},
$$

for every $x^{*} \in \mathscr{B}^{*}$, where $\mathscr{B}^{*}$ denotes the dual space of $\mathscr{B}$.

P r o o f. Let $B: \mathscr{B}^{*} \rightarrow \mathscr{B}$ be a symmetric positive operator. Then, there exists a Hilbert space $H$, and a linear continuous operator $A: \mathscr{B}^{*} \rightarrow H$ such that $B=A^{*} \cdot A$, and $\overline{A\left(\mathscr{B}^{*}\right)}=H, A^{*}(H) \subset \mathscr{B}$.

It is known that $H$ is a separable Hilbert space if $\mathscr{B}$ is a separable one (see [41]). Let $B: \mathscr{B}^{*} \rightarrow \mathscr{B}$ be the covariance operator of the random element $\xi$, then

$$
\mathbf{E}\left\langle\xi, x^{*}\right\rangle\left\langle\xi, y^{*}\right\rangle=\left\langle B x^{*}, y^{*}\right\rangle=\left\langle A x^{*}, A y^{*}\right\rangle .
$$

For all $h \in H$ of the form $h=A x^{*}$, we define a random variable

$$
\eta(h):=\left\langle\xi, x^{*}\right\rangle .
$$


Note that $\eta(h)$, for $h \in H$, is a centered Gaussian random variable with

$$
\mathbf{E} \eta^{2}(h)=\left\langle A x^{*}, A x^{*}\right\rangle=\left\|A x^{*}\right\|_{H}^{2}=\|h\|_{H}^{2},
$$

that is

$$
\eta(h) \sim N\left(0,\|h\|_{H}^{2}\right), h \in A\left(\mathscr{B}^{*}\right) .
$$

The map $h \rightarrow \eta(h), h \in A\left(\mathscr{B}^{*}\right) \subset H, \eta(h) \in L^{2}(\Omega, \mathscr{F}, \mathbf{P})$, can be extended by continuity to the isometry between $H=\overline{A\left(\mathscr{B}^{*}\right)}$ and the closure of the set of random variables $\left\{\eta(h), h \in A\left(\mathscr{B}^{*}\right)\right\}$ in $L^{2}(\Omega, \mathscr{F}, \mathbf{P})$. Thus, $\{\eta(h): h \in H\}$ is an isonormal process. Theorem 4.1 is proved.

$\mathrm{R}$ e $\mathrm{m}$ a r k 4.1 . If $\operatorname{supp} \xi \neq \mathscr{B}$, then $\widetilde{\mathscr{B}}=\operatorname{supp} \xi \subset \mathscr{B}$ is a separable Banach space, and Theorem 4.1 can be reformulated for this separable Banach space $\widetilde{\mathscr{B}}$.

Let now $\mathscr{B}=H_{0}$ be a separable Hilbert space, and let $B: H_{0} \rightarrow H_{0}$ be a symmetric positive operator. By Theorem 4.1, there exists a Hilbert space $H$ and a linear and continuous operator $A: H_{0} \rightarrow H$ such that $A^{*} A=B$. One can choose $H=H_{0}$, and $A: H_{0} \rightarrow H_{0}$ identical to $B^{1 / 2}$, the positive square root of $B$. Note that $B^{1 / 2}\left(H_{0}\right)$ is dense in $H_{0}$ (see [41]).

In a similar way to (4.1), we obtain that if $B$ is the covariance operator of the random element $\xi$ on the separable Hilbert space $H_{0}$, then, for $x, y \in H_{0}$

$$
\mathbf{E}\langle\xi, x\rangle_{H_{0}}\langle\xi, y\rangle_{H_{0}}=\langle B x, y\rangle_{H_{0}}=\left\langle B^{1 / 2} x, B^{1 / 2} y\right\rangle_{H_{0}}=\langle h, g\rangle_{H_{0}} .
$$

Then,

$$
\begin{aligned}
\mathbf{E}\left[\eta\left(h_{1}\right) \eta\left(h_{2}\right)\right] & =\mathbf{E}\left\langle\xi, x_{1}\right\rangle_{H_{0}}\left\langle\xi, x_{2}\right\rangle_{H_{0}} \\
& =\left\langle B x_{1}, x_{2}\right\rangle_{H_{0}}=\left\langle B^{1 / 2} x_{1}, B^{1 / 2} x_{2}\right\rangle_{H_{0}}=\left\langle h_{1}, h_{2}\right\rangle_{H_{0}} .
\end{aligned}
$$

As above, the map $h \rightarrow \eta(h), h \in B^{1 / 2}\left(H_{0}\right) \subset H_{0}, \eta(h) \in L^{2}(\Omega, \mathscr{F}, \mathbf{P})$, can be extended by continuity to the isometry between $H_{0}=\overline{B^{1 / 2}\left(H_{0}\right)}$ and the closure of the set of random variables $\left\{\eta(h), h \in B^{1 / 2}\left(H_{0}\right)\right\}$ in $L^{2}(\Omega, \mathscr{F}, \mathbf{P})$. Thus, $\left\{\eta(h): h \in H_{0}\right\}$ is an isonormal process.

As in Remark 4.1, if $\operatorname{supp} \xi \neq H_{0}$, then $\widetilde{H}_{0}=\operatorname{supp} \xi \subset H_{0}$ is a separable Hilbert space, and Theorem 4.1 can be reformulated for $\widetilde{H}_{0}$.

Let $\mathscr{B}$ be a real separable Banach space, and let $\xi$ be a centered Gaussian element in $\mathscr{B}$. We denote $\mathscr{H}_{0}(\xi)=\mathbf{R}^{1}, \mathscr{H}_{1}(\xi)$ a closed linear subspace of a set of r.v.'s $\left\{\left\langle\xi, x^{*}\right\rangle, x^{*} \in \mathscr{B}^{*}\right\}$ in the Hilbert space $L^{2}(\Omega, \mathscr{F}, \mathbf{P})$. Let $\mathscr{P}_{n}(\xi), n \geqslant$ 2 , be a set of polynomials of degree at least $n$ of r.v.'s $\left\langle\xi, x_{1}^{*}\right\rangle, \ldots,\left\langle\xi, x_{n}^{*}\right\rangle$, $x_{1}^{*}, \ldots, x_{n}^{*} \in \mathscr{B}^{*}$.

Denote by $\mathscr{H}_{n}(\xi)$ the closure of the linear subspace $\left\{\mathscr{P}_{n}(\xi)\right\} \ominus\left\{\mathscr{H}_{0}(\xi) \oplus\right.$ $\left.\cdots \oplus \mathscr{H}_{n-1}(\xi)\right\}, n \geqslant 2$. The set of random variables $\mathscr{H}_{n}(\xi), n \geqslant 1$, is said to be the $n$th Wiener chaos associated with $\xi$.

Proposition 4.1. Suppose that there exists an isonormal process $\eta=$ $\{\eta(h), h \in H\}$ such that $\left\langle\xi, x^{*}\right\rangle \in\{\eta(h), h \in H\}$, for every $x^{*} \in \mathscr{B}^{*}$. Then $\mathscr{H}_{n}(\xi) \subset \mathscr{H}_{n}(\eta)$, for every $n \geqslant 1$. 
$\mathrm{R}$ e $\mathrm{m}$ a $\mathrm{r}$ 4.2. Let $\{\eta(h), h \in H\}$ be the isonormal process considered in Theorem 4.1. Then, from (4.2), we obtain that

$$
\mathscr{H}_{n}(\xi)=\mathscr{H}_{n}(\eta), \quad n \geqslant 1
$$

The following proposition (see [19]) is a reformulation of Theorem 1 of [30] (see also [29]).

Proposition 4.2. Let $\{\eta(h), h \in H\}$ be an isonormal process. Consider the natural numbers $1 \leqslant n_{1}<n_{2}<\cdots<n_{K}<\infty, K \geqslant 2$, and the set of r.v.' $s \pi_{j, n_{k}}(\eta) \in \mathscr{H}_{n_{k}}(\eta), k=1, \ldots, K, j \geqslant 0$, such that

(a)

$$
\lim _{j \rightarrow \infty} \mathbf{E} \pi_{j, n_{k}}^{2}(\eta)=1, \quad k=1, \ldots, K
$$

(b)

$$
\lim _{j \rightarrow \infty} \mathbf{E} \pi_{j, n_{k}}(\eta) \pi_{j, n_{p}}(\eta)=0, \quad k \neq p, \quad k, p \in\{1, \ldots, K\}
$$

(c)

$$
\lim _{j \rightarrow \infty} \mathbf{E} \pi_{j, n_{k}}^{4}(\eta)=3, \quad k=1, \ldots, K
$$

Then, the vector $\pi_{j, K}(\eta)=\left(\pi_{j, n_{1}}(\eta), \ldots, \pi_{j, n_{K}}(\eta)\right)$ converges in distribution, as $j \rightarrow \infty$, to a multidimensional standard Gaussian vector $\pi_{K} \sim N\left(0, \mathbf{I}_{K}\right)$.

The proof follows from Lemma 4.1 .

Propositions 4.1 and 4.2 are now applied to obtain the following result.

Theorem 4.2. Let $\xi$ and $\eta$ be as in Proposition 4.1. If $\pi_{j, n_{k}}(\xi) \in$ $\mathscr{H}_{n_{k}}(\xi)$ such that

(a)

$$
\lim _{j \rightarrow \infty} \mathbf{E} \pi_{j, n_{k}}^{2}(\xi)=1, \quad k=1, \ldots, K
$$

(b)

$$
\lim _{j \rightarrow \infty} \mathbf{E} \pi_{j, n_{k}}(\xi) \pi_{j, n_{p}}(\xi)=0, \quad k \neq p, \quad k, p \in\{1, \ldots, K\}
$$

(c)

$$
\lim _{j \rightarrow \infty} \mathbf{E} \pi_{j, n_{k}}^{4}(\xi)=3, \quad k=1, \ldots, K
$$

Then, the vector

$$
\pi_{j, K}(\xi)=\left(\pi_{j, n_{1}}(\xi), \ldots, \pi_{j, n_{K}}(\xi)\right)
$$

converges in distribution, as $j \rightarrow \infty$, to a $K$-dimensional Gaussian distribution $\pi_{K} \sim N\left(0, \mathbf{I}_{K}\right)$. 
Obviously, for every $x \in \mathbf{R}_{+}^{d}, H_{n}(\xi(x)) \in \mathscr{H}_{n}(\xi)$, with $n \geqslant 1$, under the assumption of mean-square continuity of the centered Gaussian random field $\xi$.

Lemma 4.2. Let $\varphi \in C\left(\mathbf{R}_{+}^{d}\right)$, then, for $j>0$,

$$
S_{n}(j)=\int_{\left[0,2^{j}\right]^{d}} \varphi(\mathbf{x}) H_{n}(\xi(\mathbf{x})) d \mathbf{x} \in \mathscr{H}_{n}(\xi), \quad n \geqslant 1 .
$$

P r o o f. For a fixed $j>0$, and $n \geqslant 1$, and $0 \leqslant x_{0}^{k}<x_{1}^{k}<\cdots<x_{N}^{k}=$ $2^{j}, \Delta x_{i}^{k}=x_{i+1}^{k}-x_{i}^{k}=2^{j} /(N)^{1 / d}$, for $k=1, \ldots, d$, and $i=0, \ldots, N-1$. It is easy to see that, for every $N \geqslant 1$,

$$
\Sigma_{n}(N, j)=\sum_{i=0}^{N-1} \varphi\left(\mathbf{x}_{i}\right) H_{n}\left(\xi\left(\mathbf{x}_{i}\right)\right) \Delta \mathbf{x}_{i} \in \mathscr{H}_{n}(\xi) .
$$

We have

$$
\begin{aligned}
& \mathbf{E}\left(S_{n}(j)-\Sigma_{n}(N, j)\right)^{2} \\
& \leqslant 2 N \sum_{i=0}^{N-1} \mathbf{E}\left(\int_{\prod_{k=1}^{d}\left[x_{i}^{k}, x_{i+1}^{k}\right]} \varphi(\mathbf{x})\left[H_{n}(\xi(\mathbf{x}))-H_{n}\left(\xi\left(\mathbf{x}_{i}\right)\right)\right] d \mathbf{x}\right)^{2} \\
&+2 N \sum_{i=0}^{N-1} \mathbf{E}\left(\int_{\prod_{k=1}^{d}\left[x_{i}^{k}, x_{i+1}^{k}\right]}\left(\varphi(\mathbf{x})-\varphi\left(\mathbf{x}_{i}\right)\right) H_{n}\left(\xi\left(\mathbf{x}_{i}\right)\right) d \mathbf{x}\right)^{2} \\
&= \Sigma_{1}(j)+\Sigma_{2}(j) .
\end{aligned}
$$

By the continuity of the covariance function of $\xi$, for every $\varepsilon>0$, there exists $N_{0}$ such that, for $N>N_{0}$,

$$
\max _{\mathbf{x} \in \prod_{k=1}^{d}\left[x_{i}^{k}, x_{i+1}^{k}\right]} \mathbf{E}\left[H_{n}(\xi(\mathbf{x}))-H_{n}\left(\xi\left(\mathbf{x}_{i}\right)\right)\right]^{2}<\varepsilon, \quad i=0, \ldots, N-1 .
$$

If

$$
\max _{\mathbf{x} \in \prod_{k=1}^{d}\left[x_{i}^{k}, x_{i+1}^{k}\right]}\left|\varphi(\mathbf{x})-\varphi\left(\mathbf{x}_{i}\right)\right|<\varepsilon, \quad i=0, \ldots, N-1,
$$

then

$$
\Sigma_{1}(j) \leqslant 2 \max _{\mathbf{x} \in\left[0,2^{j}\right]^{d}} \varphi^{2}(\mathbf{x}) 2^{2 d j} \varepsilon, \quad \Sigma_{2}(j) \leqslant 2 n ! 2^{2 j d} \varepsilon^{2} .
$$

Thus,

$$
\lim _{N \rightarrow \infty} \mathbf{E}\left(S_{n}(j)-\Sigma_{n}(N, j)\right)^{2}=0,
$$

and hence, $S_{n}(j) \in \mathscr{H}_{n}(\xi), n \geqslant 1$, for every $j>0$. Lemma 4.2 is proved.

From Theorem 4.1 and Lemma 4.2, we obtain the following corollary. 
Corollary 4.1. If $\varphi_{j, k} \in C\left(\mathbf{R}_{+}^{d}\right), k=1, \ldots, K, j>0$, and conditions (a) and (c) of Theorem 4.2 are satisfied for r.v.'s

$$
\pi_{j, n_{k}}(\xi)=\int_{\left[0,2^{j}\right]^{d}} \varphi_{j, k}(\mathbf{x}) H_{n_{k}}(\xi(\mathbf{x})) d \mathbf{x}
$$

then the vector

$$
\pi_{j, K}(\xi)=\left(\int_{\left[0,2^{j}\right]^{d}} \varphi_{j, 1}(\mathbf{x}) H_{n_{1}}(\xi(\mathbf{x})) d \mathbf{x}, \ldots, \int_{\left[0,2^{j}\right]^{d}} \varphi_{j, K}(\mathbf{x}) H_{n_{K}}(\xi(\mathbf{x})) d \mathbf{x}\right)
$$

converges in distributions, as $j \rightarrow \infty$, to a standard Gaussian vector $\pi_{K} \sim$ $N\left(0, \mathbf{I}_{K}\right)$.

Indeed, for random variables (4.11), condition (b) of Theorem 4.2 is satisfied by the orthogonality property of Hermite polynomials.

Lemma 4.3. (i) Under condition A.1, the wavelet random field can be identified with an isonormal process $\eta_{X_{W}^{N}}$;

(ii) for the normalized wavelet random field $\left\{X_{W}^{N}(j, b), b \in L_{j}, j \in \mathbf{Z}\right\}$, given by

$$
X_{W}^{N}(j, u)=\frac{X\left(\boldsymbol{\Psi}_{j, u}\right)}{\left[B_{X}^{\alpha_{0}}\left(\boldsymbol{\Psi}_{j, u}, \mathbf{\Psi}_{j, u}\right)\right]^{1 / 2}}, \quad u \in L_{j},
$$

the integral functionals $\int_{L_{j}} \varphi(b) H_{k}\left(X_{W}^{N}(b)\right) d b$ belong to the Wiener chaos of $\eta_{X_{W}^{N}}$, for $\varphi \in C\left(\mathbf{R}_{+}^{d}\right)$.

P r o o f. (i) The proof follows by applying Theorem 4.1 with $\xi=\tilde{X}$ and $H=L^{2}(D)=\overline{s p}^{L^{2}(D)}\left\{\boldsymbol{\Psi}_{j, b}, b \in L_{j} \cap \mathbf{Z}^{d} ; j \in \mathbf{Z}\right\}$. The isonormal process $\eta$ is defined as

$$
\eta(h)=X(f)=\int_{D} f(x) \tilde{X}(x) d x
$$

where $h=\mathscr{T}_{X}(f)$, with

$$
R_{X}(f)(g)=B_{X}(f, g)=\left\langle\mathscr{T}_{X}(f), \mathscr{T}_{X}(g)\right\rangle_{L^{2}(D)} \quad \forall f, g \in L^{2}(D) .
$$

Here, $B_{X}$ is defined as in equation (2.4).

From equation (2.6), the wavelet random field is given by

$$
\begin{aligned}
X\left(\boldsymbol{\Psi}_{j, b}\right) & =\int_{D} \tilde{X}(x) \boldsymbol{\Psi}_{j, b}(x) d x=\left\langle\tilde{X}, \boldsymbol{\Psi}_{j, b}\right\rangle_{L^{2}(D)} \\
& =\eta\left(\mathscr{T}_{X}\left(\mathbf{\Psi}_{j, b}\right)\right), \quad b \in L_{j} \subset \mathbf{R}_{+}^{d},
\end{aligned}
$$

which proves the first assertion.

(ii) Finally, since the $n$th Wiener chaos $\mathscr{H}_{n}$ is defined as the closed linear subspace, in $L^{2}(\Omega, \mathscr{F}, \mathbf{P})$, generated by the random variables 
$\left\{H_{n}(\eta(h)), h \in H,\|h\|_{H}=1\right\}$, in our case, from equation (4.13),

$$
\begin{aligned}
X_{W}^{N}(j, b) & =\left\langle\tilde{X}, \frac{\boldsymbol{\Psi}_{j, b}}{\left[B_{X}^{\alpha_{0}}\left(\boldsymbol{\Psi}_{j, b}, \mathbf{\Psi}_{j, b}\right)\right]^{1 / 2}}\right\rangle_{L^{2}(D)} \\
& =\eta\left(\mathscr{T}_{X}^{\alpha_{0}}\left(\frac{\mathbf{\Psi}_{j, b}}{\left[B_{X}^{\alpha_{0}}\left(\mathbf{\Psi}_{j, b}, \boldsymbol{\Psi}_{j, b}\right)\right]^{1 / 2}}\right)\right)
\end{aligned}
$$

with

$$
\left\|\mathscr{T}_{X}^{\alpha_{0}}\left(\frac{\boldsymbol{\Psi}_{j, b}}{\left[B_{X}^{\alpha_{0}}\left(\mathbf{\Psi}_{j, b}, \mathbf{\Psi}_{j, b}\right)\right]^{1 / 2}}\right)\right\|_{L^{2}(D)}=1
$$

due to the fact

$$
\left\|\mathscr{T}_{X}^{\alpha_{0}}\left(\boldsymbol{\Psi}_{j, b}\right)\right\|_{L^{2}(D)}=\left[B_{X}^{\alpha_{0}}\left(\boldsymbol{\Psi}_{j, b}, \boldsymbol{\Psi}_{j, b}\right)\right]^{1 / 2}
$$

Then, from Lemma 4.2 the second assertion follows. Lemma 4.3 is proved.

$\mathrm{R}$ e $\mathrm{m}$ a r k 4.3. Note that, as we have assumed that the discrete version provided by $\left\{\boldsymbol{\Psi}_{j, b}, \quad b \in L_{j} \cap \mathbf{Z}^{d}, j \in \mathbf{Z}\right\}$ of our CDWT coincides with the continuous one over the nodes of the dyadic regular grid defined by discrete translations at each scale, the following identities then hold:

$L^{2}(D)=\overline{s p}^{L^{2}(D)}\left\{\boldsymbol{\Psi}_{j, b}, b \in L_{j} \cap \mathbf{Z}^{d}, j \in \mathbf{Z}\right\} \subseteq \overline{s p}^{L^{2}(D)}\left\{\boldsymbol{\Psi}_{j, b}, b \in L_{j}, j \in \mathbf{Z}\right\}$.

Furthermore,

$$
\left\langle\boldsymbol{\Psi}_{j, b}, \boldsymbol{\Psi}_{l, u}\right\rangle_{L^{2}(D)}=\delta_{j, l} \delta(b-u), \quad b, u \in L_{j} \cap \mathbf{Z}^{d}, \quad j, l \in \mathbf{Z}
$$

where $\int_{D} \delta(b-u) h(u) d u=h(b)$. Therefore, for every function $f \in L^{2}(D)$,

$$
f(x)=\sum_{j} \int_{L_{j}} f_{j, b} \boldsymbol{\Psi}_{j, b}(x) d b,
$$

and

$$
X(f)=\sum_{j} \int_{L_{j}} f_{j, b} X\left(\mathbf{\Psi}_{j, b}\right) d b=\sum_{j} \int_{L_{j}} f_{j, b} \eta\left(g_{j}(b)\right) d b,
$$

where $g_{j}(b)=\mathscr{T}_{X}\left(\boldsymbol{\Psi}_{j, b}\right)$.

In the case where the RKHS of $X$ is isomorphic to a fractional Sobolev space $H^{\alpha_{0}}(D)$, we have that operators $\mathscr{T}_{X}: \bar{H}^{-\alpha_{0}}(D) \longrightarrow L^{2}(D)$ and $\mathscr{T}_{X}^{*}$ : $L^{2}(D) \longrightarrow H^{\alpha_{0}}(D)$ define isomorphisms. The isonormal process $\eta$ is then defined, as before, on $\mathscr{T}_{X}\left(\bar{H}^{-\alpha_{0}}(D)\right)=L^{2}(D)$. The covariance function $B_{X}$ provides the following inner product:

$$
\langle f, g\rangle_{B_{X}}=B_{X}(f, g)=\left\langle\mathscr{T}_{X}(f), \mathscr{T}_{X}(g)\right\rangle_{L^{2}(D)} \quad \forall f, g \in \bar{H}^{-\alpha_{0}}(D),
$$


on the space $\bar{H}^{-\alpha_{0}}(D)$, which generates an equivalent norm to the original one associated with the fractional Sobolev space $\bar{H}^{-\alpha_{0}}(D)$. Thus,

$$
[\mathscr{H}(X)]^{*} \equiv\left(\bar{H}^{-\alpha_{0}}(D),\langle\cdot, \cdot\rangle_{B_{X}}\right) \simeq\left(\bar{H}^{-\alpha_{0}}(D),\langle\cdot, \cdot\rangle_{\bar{H}^{-\alpha_{0}}(D)}\right),
$$

where $\simeq$ and $\equiv$, respectively, represent the isomorphic and the isometrical equivalences. Here, as before, $[\mathscr{H}(X)]^{*}$ denotes the dual of the RKHS space $\mathscr{H}(X)$ of $X$. Therefore, the generalized random field $X$ can also be identified with an isonormal process on the Hilbert space $H=[\mathscr{H}(X)]^{*} \equiv$ $\left(\bar{H}^{-\alpha_{0}}(D),\langle\cdot, \cdot\rangle_{B_{X}}\right)$.

5. Asymptotic normality. This section is devoted to the derivation of our main result, Theorem 5.1, on the asymptotic Gaussian distribution of the minimum contrast estimator given in (3.11). The key previous results are Lemmas 4.1 and 4.3. Specifically, Lemma 4.3 allows the application of Corollary 4.1, obtained from Lemma 4.1, in the derivation of the proof of Theorem 5.1.

Different methodologies have been used for obtaining the asymptotic normality of minimum contrast estimators defined in the spectral domain. For instance, we could mention the limit result derived in [14] for nonstationary Gaussian processes with stationary increments, with convergence rate $\sqrt{N}$, being $N$ the number of observations. Similarly, in [20], for the case of continuous observations on an interval $[0, T]$, the asymptotic normality of the minimum contrast parameter estimator defined for random processes and fields is obtained with convergence rate $T^{d / 2}$, where $d$ denotes the spatial dimension, in particular, $d=1$ for the random process case (see also [4]).

In our result, the rate of convergence obtained is of order $j^{-1}$, due to the fact that the integral wavelet functionals involved in the proof of the main central limit result are defined from the derivative of the contrast process, which involves the partial derivative with respect to the parameter $\alpha$ of the asymptotic variance of the wavelet random field. This partial derivative is directly proportional to $j$. The divergence order of the asymptotic variance of these wavelet functionals is then of order $j^{2}$. Note that parameter $j$ defines the exponent that determines the dyadic potential increasing rate of the translation parameter domain, with respect to which our asymptotic approach is defined.

Theorem 5.1. Under conditions A.1-A.4 and B.1-B.2, as $j \rightarrow \infty$,

$$
\kappa(j)\left(\widetilde{\alpha}_{j}-\alpha_{0}\right) \longrightarrow N\left(0, \Gamma\left(\alpha_{0}\right)\right)
$$

in distribution, where $\kappa(j)=1 / j$, and

$$
\Gamma\left(\alpha_{0}\right)=\nu\left(\alpha_{0}\right)^{-2}\left[\sum_{k=1}^{\infty} \frac{C_{k}^{2}}{k !} \sigma_{k}^{2}\left(\alpha_{0}\right)\right],
$$




$$
\nu\left(\alpha_{0}\right)=\lim _{j \rightarrow \infty} \int_{L_{j}} \omega(b) \frac{\partial^{2}}{\partial \alpha^{2}}\left[\ln B_{X}^{\alpha}\left(\boldsymbol{\Psi}_{j, b}, \mathbf{\Psi}_{j, b}\right)\right]\left(\alpha_{0}\right) d b
$$

with

$$
\begin{aligned}
\sigma_{k}^{2}\left(\alpha_{0}\right)=4\left[\ln \frac{1}{2}\right]^{2}\left[\int_{\mathbf{R}^{d} \backslash \mathscr{B}_{R}^{d}(0)} \frac{\|x\|^{k\left(2 \alpha_{0}-3 d\right)}}{\left[\widetilde{L}_{d}\left(\alpha_{0}\right)\right]^{k}} \omega * \omega(x) d x\right. \\
\left.+\frac{1}{\left[2 \alpha_{0}\left(2 \alpha_{0}+d\right)\right]^{k}} \int_{\mathscr{B}_{R}^{d}(0)} \frac{\left[\xi_{v}(x)\right]^{k}}{\left[\widetilde{L}_{d}\left(\alpha_{0}\right)\right]^{k}} \omega * \omega(x) d x\right],
\end{aligned}
$$

for $k \geqslant 1$, where $\mathscr{B}_{R}^{d}(0)$ denotes the ball of center zero and radius $R$, with $R \gg 1$ and $\xi_{v}$ and $\widetilde{L}_{d}\left(\alpha_{0}\right)$ are given by (3.6) and (3.7), respectively. Here

$$
\omega * \omega(x)=\int_{D(x)} \omega(b) \omega(b-x) d b
$$

with $D(x)=\left\{b \in \mathbf{R}_{+}^{d}, b_{i}>x_{i}, i=1, \ldots, d\right\}$, for $x=\left(x_{1}, \ldots, x_{d}\right)$ and $b=\left(b_{1}, \ldots, b_{d}\right)$.

P r o of of The or e m 5.1. The proof of Theorem 5.1 has two parts. The first part follows from Lemma 4.3 and Corollary 4.1, and it provides the convergence to the Gaussian distribution of a finite-dimensional sum of integral Hermite functionals of the normalized wavelet random field. The second part (see Lemma 5.1) shows the convergence to the Gaussian distribution of the infinite-dimensional integral Hermite series defining the partial derivative of the contrast process with respect to parameter $\alpha$.

First part: Convergence of the finite-dimensional Hermite sum. We now proceed to develop the first part of the proof of Theorem 5.1.

For $j$ sufficiently large, the following identity holds:

$$
\left[\frac{\partial}{\partial \alpha} U_{j}\right]\left(\widetilde{\alpha}_{j}\right)-\left[\frac{\partial}{\partial \alpha} U_{j}\right]\left(\alpha_{0}\right)=\left[\frac{\partial^{2}}{\partial \alpha^{2}} U_{j}\right]\left(\alpha_{j}^{*}\right)\left(\widetilde{\alpha}_{j}-\alpha_{0}\right),
$$

for $\alpha_{j}^{*}$ in the interval $\left(\alpha_{0}, \widetilde{\alpha}_{j}\right)$. Equivalently,

$$
-\left[\frac{\partial}{\partial \alpha} U_{j}\right]\left(\alpha_{0}\right)=\left[\frac{\partial^{2}}{\partial \alpha^{2}} U_{j}\right]\left(\alpha_{j}^{*}\right)\left(\widetilde{\alpha}_{j}-\alpha_{0}\right), \quad \alpha_{j}^{*} \in\left(\alpha_{0}, \widetilde{\alpha}_{j}\right) .
$$

Furthermore, from Lemmas 3.2 and 3.3,

$$
-\left[\frac{\partial^{2}}{\partial \alpha^{2}} U_{j}\right]\left(\alpha_{j}^{*}\right) \longrightarrow \nu\left(\alpha_{0}\right) \text { in probability, }
$$

as $j \rightarrow \infty$, where $\nu\left(\alpha_{0}\right)$ is defined as in equation (5.2). Therefore, from Slutskii's lemma, in order to prove that $\kappa(j)\left(\widetilde{\alpha}_{j}-\alpha_{0}\right) \longrightarrow N\left(0, \Gamma\left(\alpha_{0}\right)\right)$, it is 
sufficient to show that

$$
\begin{aligned}
\kappa(j)\left[\frac{\partial}{\partial \alpha} U_{j}\right]\left(\alpha_{0}\right)=\kappa(j) \int_{L_{j}} \frac{S(j, b, b)-\mathbf{E}_{\alpha_{0}}(S(j, b, b))}{\left[B_{X}^{\alpha_{0}}\left(\mathbf{\Psi}_{j, b}, \mathbf{\Psi}_{j, b}\right)\right]^{2}} \\
\quad \times\left[\frac{\partial}{\partial \alpha} B_{X}^{\alpha}\left(\mathbf{\Psi}_{j, b}, \mathbf{\Psi}_{j, b}\right)\right]\left(\alpha_{0}\right) \omega(b) d b \longrightarrow N\left(0, G\left(\alpha_{0}\right)\right),
\end{aligned}
$$

in distribution, where

$$
G\left(\alpha_{0}\right)=\nu\left(\alpha_{0}\right)^{2} \Gamma\left(\alpha_{0}\right)=\sum_{k=1}^{\infty} \frac{C_{k}^{2}}{k !} \sigma_{k}^{2}\left(\alpha_{0}\right)
$$

with $\sigma_{k}^{2}\left(\alpha_{0}\right)$ being defined as in equation (5.3), for each $k \geqslant 1$.

For the derivation of limit (5.6), consider the process $Z_{j}\left(\alpha_{0}\right)$ given by

$$
Z_{j}\left(\alpha_{0}\right)=\int_{L_{j}}\left[\frac{\partial}{\partial \alpha} B_{X}^{\alpha}\left(\boldsymbol{\Psi}_{j, b}, \mathbf{\Psi}_{j, b}\right)\right]\left(\alpha_{0}\right) \frac{S(j, b, b)}{\left[B_{X}^{\alpha_{0}}\left(\mathbf{\Psi}_{j, b}, \mathbf{\Psi}_{j, b}\right)\right]^{2}} \omega(b) d b,
$$

with expected value

$$
\mathbf{E}\left(Z_{j}\left(\alpha_{0}\right)\right)=\int_{L_{j}}\left[\frac{\partial}{\partial \alpha} B_{X}^{\alpha}\left(\boldsymbol{\Psi}_{j, b}, \boldsymbol{\Psi}_{j, b}\right)\right]\left(\alpha_{0}\right)\left[B_{X}^{\alpha_{0}}\left(\boldsymbol{\Psi}_{j, b}, \boldsymbol{\Psi}_{j, b}\right)\right]^{-1} \omega(b) d b .
$$

For the application of Lemma 4.1 , process $Z_{j}\left(\alpha_{0}\right)$ is rewritten as

$$
\begin{aligned}
Z_{j}\left(\alpha_{0}\right)=\int_{L_{j}} & \omega(b)\left[\frac{\partial}{\partial \alpha} B_{X}^{\alpha}\left(\mathbf{\Psi}_{j, b}, \mathbf{\Psi}_{j, b}\right)\right]\left(\alpha_{0}\right)\left[B_{X}^{\alpha_{0}}\left(\boldsymbol{\Psi}_{j, b}, \mathbf{\Psi}_{j, b}\right)\right]^{-1} \\
& \times F\left(\frac{X\left(\mathbf{\Psi}_{j, b}\right)}{\left[B_{X}^{\alpha_{0}}\left(\mathbf{\Psi}_{j, b}, \mathbf{\Psi}_{j, b}\right)\right]^{1 / 2}}\right) d b
\end{aligned}
$$

in terms of the functional

$$
F\left(\frac{X\left(\mathbf{\Psi}_{j, u}\right)}{\left[B_{X}^{\alpha_{0}}\left(\mathbf{\Psi}_{j, u}, \mathbf{\Psi}_{j, u}\right)\right]^{1 / 2}}\right)=\frac{\left|X\left(\boldsymbol{\Psi}_{j, u}\right)\right|^{2}}{B_{X}^{\alpha_{0}}\left(\boldsymbol{\Psi}_{j, u}, \mathbf{\Psi}_{j, u}\right)}=\frac{S(j, u, u)}{\left[B_{X}^{\alpha_{0}}\left(\boldsymbol{\Psi}_{j, u}, \mathbf{\Psi}_{j, u}\right)\right]}
$$

of the normalized wavelet random field $\left\{X_{W}^{N}(j, b), b \in L_{j}, j \in \mathbf{Z}\right\}$, given by

$$
X_{W}^{N}(j, u)=\frac{X\left(\boldsymbol{\Psi}_{j, u}\right)}{\left[B_{X}^{\alpha_{0}}\left(\boldsymbol{\Psi}_{j, u}, \boldsymbol{\Psi}_{j, u}\right)\right]^{1 / 2}}, \quad u \in L_{j} .
$$

The Hermite expansion of $F$ then leads to the following series expansion of process $Z_{j}\left(\alpha_{0}\right)$ :

$$
Z_{j}\left(\alpha_{0}\right)=\sum_{k=0}^{\infty} \frac{C_{k}}{k !} \eta_{k}^{\alpha_{0}}\left(X_{W}^{N}\right)(j)
$$

where

$$
\begin{aligned}
\eta_{k}^{\alpha_{0}}\left(X_{W}^{N}\right)(j)= & \int_{L_{j}} \omega(b)\left[\frac{\partial}{\partial \alpha} B_{X}^{\alpha}\left(\boldsymbol{\Psi}_{j, b}, \boldsymbol{\Psi}_{j, b}\right)\right]\left(\alpha_{0}\right) \\
& \times\left[B_{X}^{\alpha_{0}}\left(\boldsymbol{\Psi}_{j, b}, \mathbf{\Psi}_{j, b}\right)\right]^{-1} H_{k}\left(X_{W}^{N}(j, b)\right) d b .
\end{aligned}
$$


For $k \geqslant 1$, considering the isometry between the $k$ th Wiener chaos associated with the generalized random field $X$, and the $k$ th symmetric tensor product $H^{\odot k}$ of $H$, defined by the multiple Wiener-Itô stochastic integral $I_{k}^{X}$ with respect to the process $X$, the following identity then holds:

$$
\begin{aligned}
\eta_{k}^{\alpha_{0}}\left(X_{W}^{N}\right)(j)=I_{k}^{X}\left(f_{j}^{\alpha_{0}}\right) \\
=\int_{D^{k}}\left[\int_{L_{j}} \omega(b)\left[\frac{\partial}{\partial \alpha} B_{X}^{\alpha}\left(\mathbf{\Psi}_{j, b}, \mathbf{\Psi}_{j, b}\right)\right]\left(\alpha_{0}\right)\left[B_{X}^{\alpha_{0}}\left(\mathbf{\Psi}_{j, b}, \mathbf{\Psi}_{j, b}\right)\right]^{-1}\right. \\
\left.\times \prod_{l=1}^{k} \frac{\mathbf{\Psi}_{j, b}\left(x_{l}\right)}{\left[B_{X}^{\alpha_{0}}\left(\mathbf{\Psi}_{j, b}, \mathbf{\Psi}_{j, b}\right)\right]^{1 / 2}} d b\right] \prod_{l=1}^{k} \widetilde{X}\left(x_{l}\right) d x_{l} \\
=\int_{D^{k}}\left[\int_{L_{j}} \omega(b)\left[\frac{\partial}{\partial \alpha} B_{X}^{\alpha}\left(\mathbf{\Psi}_{j, b}, \mathbf{\Psi}_{j, b}\right)\right]\left(\alpha_{0}\right)\left[B_{X}^{\alpha_{0}}\left(\mathbf{\Psi}_{j, b}, \mathbf{\Psi}_{j, b}\right)\right]^{-1}\right. \\
\left.\quad \times \prod_{l=1}^{k} \mathscr{T}_{X}\left(\frac{\mathbf{\Psi}_{j, b}}{\left[B_{X}^{\alpha_{0}}\left(\mathbf{\Psi}_{j, b}, \mathbf{\Psi}_{j, b}\right)\right]^{1 / 2}}\right)\left(x_{l}\right) d b\right] \prod_{l=1}^{k} \eta\left(d x_{l}\right),(5)
\end{aligned}
$$

where operator $\mathscr{T}_{X}$ and isonormal process $\eta$ are defined as in Lemma 4.3. That is, here, $\eta(d x)$ denotes the Wiener measure. Therefore, the application of Lemma 4.1 will be performed in terms of the sequence of kernels $\left\{f_{j}^{\alpha_{0}} \in\right.$ $\left.H^{\odot k}, j \geqslant 1\right\}$, defined from the identity

$$
\begin{aligned}
f_{j}^{\alpha_{0}}\left(x_{1}, \ldots, x_{k}\right)=\int_{L_{j}} \omega(b)\left[\frac{\partial}{\partial \alpha} B_{X}^{\alpha}\left(\mathbf{\Psi}_{j, b}, \mathbf{\Psi}_{j, b}\right)\right]\left(\alpha_{0}\right)\left[B_{X}^{\alpha_{0}}\left(\mathbf{\Psi}_{j, b}, \mathbf{\Psi}_{j, b}\right)\right]^{-1} \\
\times \prod_{l=1}^{k} \mathscr{T}_{X}\left(\frac{\mathbf{\Psi}_{j, b}}{\left[B_{X}^{\alpha_{0}}\left(\mathbf{\Psi}_{j, b}, \mathbf{\Psi}_{j, b}\right)\right]^{1 / 2}}\right)\left(x_{l}\right) d b
\end{aligned}
$$

for each $k \geqslant 1$. Here, since we are interested in the asymptotic results, when $j \rightarrow \infty$, without loss of generality, parameter $j$ will move in the positive integers as in Lemma 4.1. From now on, we incorporate the scaling $\kappa(j)$ in the definition of the functional sequence $\left\{f_{j}^{\alpha_{0}}, j \geqslant 1\right\}$, i.e., we consider the sequence $\left\{\tilde{f}_{j}^{\alpha_{0}}=\kappa(j) f_{j}^{\alpha_{0}}, j \geqslant 1\right\}$.

Before applying Lemma 4.1, we compute, for $k \geqslant 1$,

$$
\lim _{j \rightarrow \infty} k !\left\|\widetilde{f}_{j}^{\alpha_{0}}\right\|_{H^{\otimes k}}^{2}=\lim _{j \rightarrow \infty} \mathbf{E}\left(I_{k}^{X}\left(\widetilde{f}_{j}^{\alpha_{0}}\right)^{2}\right) .
$$

Thus, for $k \geqslant 1$,

$$
\begin{gathered}
\mathbf{E}\left(I_{k}^{X}\left(\widetilde{f}_{j}^{\alpha_{0}}\right)^{2}\right)=k !\left\|\widetilde{f}_{j}^{\alpha_{0}}\right\|_{H^{\otimes k}}^{2} \\
=\kappa^{2}(j) k ! \int_{D^{k} \times D^{k}}\left[\int_{L_{j}} \omega(b)\left[\frac{\partial}{\partial \alpha} B_{X}^{\alpha}\left(\mathbf{\Psi}_{j, b}, \boldsymbol{\Psi}_{j, b}\right)\right]\left(\alpha_{0}\right)\left[B_{X}^{\alpha_{0}}\left(\mathbf{\Psi}_{j, b}, \mathbf{\Psi}_{j, b}\right)\right]^{-1}\right. \\
\left.\quad \times \prod_{l=1}^{k} \frac{\mathbf{\Psi}_{j, b}\left(x_{l}\right)}{\left[B_{X}^{\alpha_{0}}\left(\mathbf{\Psi}_{j, b}, \mathbf{\Psi}_{j, b}\right)\right]^{1 / 2}} d b\right]
\end{gathered}
$$




$$
\begin{aligned}
& \times\left[\int_{L_{j}} \omega(u)\left[\frac{\partial}{\partial \alpha} B_{X}^{\alpha}\left(\boldsymbol{\Psi}_{j, u}, \boldsymbol{\Psi}_{j, u}\right)\right]\left(\alpha_{0}\right)\left[B_{X}^{\alpha_{0}}\left(\boldsymbol{\Psi}_{j, u}, \boldsymbol{\Psi}_{j, u}\right)\right]^{-1}\right. \\
& \left.\times \prod_{l=1}^{k} \frac{\mathbf{\Psi}_{j, u}\left(y_{l}\right)}{\left[B_{X}^{\alpha_{0}}\left(\mathbf{\Psi}_{j, u}, \mathbf{\Psi}_{j, u}\right)\right]^{1 / 2}} d u\right] \prod_{l=1}^{k} B_{\widetilde{X}}\left(x_{l}, y_{l}\right) d x_{l} d y_{l} \\
& =\kappa^{2}(j) k ! \int_{L_{j} \times L_{j}} \omega(b)\left[\frac{\partial}{\partial \alpha} B_{X}^{\alpha}\left(\boldsymbol{\Psi}_{j, b}, \boldsymbol{\Psi}_{j, b}\right)\right]\left(\alpha_{0}\right)\left[B_{X}^{\alpha_{0}}\left(\boldsymbol{\Psi}_{j, b}, \boldsymbol{\Psi}_{j, b}\right)\right]^{-1} \\
& \times \omega(u)\left[\frac{\partial}{\partial \alpha} B_{X}^{\alpha}\left(\boldsymbol{\Psi}_{j, u}, \boldsymbol{\Psi}_{j, u}\right)\right]\left(\alpha_{0}\right)\left[B_{X}^{\alpha_{0}}\left(\boldsymbol{\Psi}_{j, u}, \boldsymbol{\Psi}_{j, u}\right)\right]^{-1} \\
& \times \frac{\left[B_{X}^{\alpha_{0}}\left(\boldsymbol{\Psi}_{j, b}, \boldsymbol{\Psi}_{j, u}\right)\right]^{k}}{\left[B_{X}^{\alpha_{0}}\left(\boldsymbol{\Psi}_{j, b}, \boldsymbol{\Psi}_{j, b}\right)\right]^{k / 2}\left[B_{X}^{\alpha_{0}}\left(\boldsymbol{\Psi}_{j, u}, \boldsymbol{\Psi}_{j, u}\right)\right]^{k / 2}} d u d b .
\end{aligned}
$$

For $j$ sufficiently large, from Lemma 3.1, equation (5.10) can be rewritten as

$$
\begin{gathered}
\mathbf{E}\left(I_{k}^{X}\left(\tilde{f}_{j}^{\alpha_{0}}\right)^{2}\right) \\
=\kappa^{2}(j) k ! \int_{L_{j} \times L_{j}} \omega(b)\left[\frac{\partial}{\partial \alpha} 2^{-2 j \alpha} C_{\boldsymbol{\Psi}}(\alpha, b, j)\right]\left(\alpha_{0}\right) 2^{2 j \alpha_{0}}\left[C_{\boldsymbol{\Psi}}\left(\alpha_{0}, b, j\right)\right]^{-1} \\
\times \omega(u)\left[\frac{\partial}{\partial \alpha} 2^{-2 j \alpha} C_{\boldsymbol{\Psi}}(\alpha, u, j)\right]\left(\alpha_{0}\right) 2^{2 j \alpha_{0}}\left[C_{\boldsymbol{\Psi}}\left(\alpha_{0}, u, j\right)\right]^{-1} \\
\times \frac{2^{-2 j k \alpha_{0}}\left[C_{\boldsymbol{\Psi}}\left(\alpha_{0}, b, u, j\right)\right]^{k}}{2^{-j \alpha_{0} k}\left[C_{\boldsymbol{\Psi}}\left(\alpha_{0}, b, j\right)\right]^{k / 2} 2^{-j \alpha_{0} k}\left[C_{\boldsymbol{\Psi}}\left(\alpha_{0}, u, j\right)\right]^{k / 2}} d u d b \\
=\kappa^{2}(j) k ! 2^{4 j \alpha_{0}} \int_{L_{j} \times L_{j}} \frac{\omega(b) \omega(u)\left[\frac{\partial}{\partial \alpha} 2^{-2 j \alpha} C_{\boldsymbol{\Psi}}(\alpha, b, j)\right]\left(\alpha_{0}\right)\left[C_{\boldsymbol{\Psi}}\left(\alpha_{0}, b, j\right)\right]^{-1}}{\times\left[C_{\boldsymbol{\Psi}}\left(\alpha_{0}, u, j\right)\right]^{-1}\left[\frac{\partial}{\partial \alpha} 2^{-2 j \alpha} C_{\boldsymbol{\Psi}}(\alpha, u, j)\right]\left(\alpha_{0}\right)} \\
\times \frac{\left[C_{\boldsymbol{\Psi}}\left(\alpha_{0}, b, u, j\right)\right]^{k}}{\left[C_{\boldsymbol{\Psi}}\left(\alpha_{0}, b, j\right)\right]^{k / 2}\left[C_{\boldsymbol{\Psi}}\left(\alpha_{0}, u, j\right)\right]^{k / 2}} d u d b,
\end{gathered}
$$

where $C_{\boldsymbol{\Psi}}\left(\alpha_{0}, b, u, j\right)$ and $C_{\boldsymbol{\Psi}}\left(\alpha_{0}, b, j\right)$ are defined as in Lemma 3.1. Moreover, from this Lemma, for $j \rightarrow \infty$,

$$
\begin{aligned}
& {\left[\frac{\partial}{\partial \alpha} 2^{-2 j \alpha} C_{\Psi}(\alpha, \infty)\right]\left(\alpha_{0}\right) \simeq 2 j 2^{-2 j \alpha_{0}} \ln (1 / 2) \widetilde{L}_{d}\left(\alpha_{0}\right)+2^{-2 j \alpha_{0}}\left[\frac{\partial}{\partial \alpha} \widetilde{L}_{d}\right]\left(\alpha_{0}\right)} \\
& =2 j 2^{-2 j \alpha_{0}} \ln \frac{1}{2} \widetilde{L}_{d}\left(\alpha_{0}\right) \\
& \quad+2^{-2 j \alpha_{0}} \frac{2[\ln 2] \alpha_{0}\left(2 \alpha_{0}+d\right) 2^{-\left(2 \alpha_{0}+d-2\right)}-\left(4 \alpha_{0}+d\right)\left[1-2^{-\left(2 \alpha_{0}+d-2\right)}\right]}{\left[\alpha_{0}\left(2 \alpha_{0}+d\right)\right]^{2}}
\end{aligned}
$$

Thus, as $j \rightarrow \infty$, the following limit expression is obtained for equation (5.11):

$\mathbf{E}\left(I_{k}^{X}\left(\tilde{f}_{j}^{\alpha_{0}}\right)^{2}\right) \rightarrow\left[2 \ln \frac{1}{2}\right]^{2} k ! \int_{\mathbf{R}_{+}^{d} \times \mathbf{R}_{+}^{d}} \omega(b) \omega(u) \frac{\left[C_{\boldsymbol{\Psi}}\left(\alpha_{0}, b-u, \infty\right)\right]^{k}}{\left[\widetilde{L}_{d}\left(\alpha_{0}\right)\right]^{k}} d u d b$ 


$$
\begin{aligned}
& =\left[2 \ln \frac{1}{2}\right]^{2} k ! \int_{\mathbf{R}^{d}} \frac{\left[C_{\mathbf{\Psi}}\left(\alpha_{0}, x, \infty\right)\right]^{k}}{\left[\widetilde{L}_{d}\left(\alpha_{0}\right)\right]^{k}} \omega * \omega(x) d x \\
& \sim 4\left[\ln \frac{1}{2}\right]^{2} k !\left[\int_{\mathbf{R}^{d} \backslash \mathscr{B}_{R}^{d}(0)} \frac{\|x\|^{k\left(2 \alpha_{0}-3 d\right)}}{\left[\widetilde{L}_{d}\left(\alpha_{0}\right)\right]^{k}} \omega * \omega(x) d x\right. \\
& \left.+\frac{1}{\left[2 \alpha_{0}\left(2 \alpha_{0}+d\right)\right]^{k}} \int_{\mathscr{B}_{R}^{d}(0)} \frac{\left[\xi_{v}(x)\right]^{k}}{\left[\widetilde{L}_{d}\left(\alpha_{0}\right)\right]^{k}} \omega * \omega(x) d x\right] \\
& =k ! \sigma_{k}^{2}\left(\alpha_{0}\right) \text {, }
\end{aligned}
$$

for $\omega \in L^{1}\left(\mathbf{R}_{+}^{d}\right)$, and with $\omega * \omega(x), x \in \mathbf{R}^{d}$, being defined as in equation (5.4). Note that

$$
\begin{aligned}
\sigma_{1}^{2}\left(\alpha_{0}\right)=4\left[\ln \frac{1}{2}\right]^{2}\left[\int_{\mathbf{R}^{d} \backslash \mathscr{B}_{R}^{d}(0)} \frac{\|x\|^{2 \alpha_{0}-3 d}}{\left[\widetilde{L}_{d}\left(\alpha_{0}\right)\right]} \omega * \omega(x) d x\right. \\
\left.+\frac{1}{\left[2 \alpha_{0}\left(2 \alpha_{0}+d\right)\right]} \int_{\mathscr{B}_{R}^{d}(0)} \frac{\left[\xi_{v}(x)\right]}{\left[\widetilde{L}_{d}\left(\alpha_{0}\right)\right]} \omega * \omega(x) d x\right]
\end{aligned}
$$

is clearly finite from the asymptotic order of function $\|x\|^{2 \alpha_{0}-3 d}$ and the continuity of function $\xi_{v}$, given in equation (3.6), as well as from the absolute integrability of function $\omega$. Moreover, for $k \geqslant 2$, from Lemma 3.1, applying Cauchy-Schwarz inequality, we have

$$
\begin{aligned}
& \lim _{j \rightarrow \infty} \mathbf{E}\left(I_{k}^{X}\left(\widetilde{f}_{j}^{\alpha_{0}}\right)^{2}\right) \leqslant 4\left[\ln \frac{1}{2}\right]^{2} k !\left[\int_{\mathbf{R}_{+}^{d} \times \mathbf{R}_{+}^{d}} \omega(b) \omega(u) d b d u\right]^{1 / 2} \\
& \times\left[\int_{\mathbf{R}_{+}^{d} \times \mathbf{R}_{+}^{d}} \omega(b) \omega(u) \frac{\left[C_{\mathbf{\Psi}}\left(\alpha_{0}, b, u, \infty\right)\right]^{2 k}}{\left[\widetilde{L}_{d}\left(\alpha_{0}\right)\right]^{2 k}} d b d u\right]^{1 / 2} \\
& \leqslant 4\left[\ln \frac{1}{2}\right]^{2} k !\|\omega\|_{L^{1}\left(\mathbf{R}_{+}^{d}\right)}\left[\int_{\mathbf{R}_{+}^{d} \times \mathbf{R}_{+}^{d}} \omega(b) \omega(u) \frac{\left[C_{\mathbf{\Psi}}\left(\alpha_{0}, b, u, \infty\right)\right]^{2}}{\left[\widetilde{L}_{d}\left(\alpha_{0}\right)\right]^{2}} d b d u\right]^{1 / 2} \\
& \sim 4\left[\ln \frac{1}{2}\right]^{2} k !\|\omega\|_{L^{1}\left(\mathbf{R}_{+}^{d}\right)} {\left[\int_{\mathbf{R}^{d} \backslash \mathscr{B}_{R}^{d}(0)} \frac{\|x\|^{4 \alpha_{0}-6 d}}{\left[\widetilde{L}_{d}\left(\alpha_{0}\right)\right]^{2}} \omega * \omega(x) d x\right.} \\
&\left.+\frac{1}{\left[2 \alpha_{0}\left(2 \alpha_{0}+d\right)\right]^{2}} \int_{\mathscr{B}_{R}^{d}(0)} \frac{\left[\xi_{v}(x)\right]^{2}}{\left[\widetilde{L}_{d}\left(\alpha_{0}\right)\right]^{2}} \omega * \omega(x) d x\right]^{1 / 2},
\end{aligned}
$$

which again is finite in view of the asymptotic order of function $\|x\|^{4 \alpha_{0}-6 d}$, and the continuity of function $\left[\xi_{v}\right]^{2}$, as well as from the absolute integrability of the weight function $\omega$.

We now proceed to check condition (ii) of Lemma 4.1. Specifically, for each $k \geqslant 2$, the contraction of order $p$, for $p=1, \ldots, k-1$, is given by

$$
\widetilde{f}_{j}^{\alpha_{0}} \otimes_{p} \widetilde{f}_{j}^{\alpha_{0}}\left(x_{1}, \ldots, x_{2 k-2 p}\right)=\int_{D^{p} \times D^{p}} \tilde{f}_{j}^{\alpha_{0}}\left(x_{1}, \ldots, x_{k-p}, y_{1}, \ldots, y_{p}\right)
$$




$$
\begin{aligned}
& \times \tilde{f}_{j}^{\alpha_{0}}\left(x_{k-p+1}, \ldots, x_{2 k-2 p}, z_{1}, \ldots, z_{p}\right) \prod_{l=1}^{p} d y_{l} d z_{l} \\
& =\kappa^{2}(j) \int_{L_{j} \times L_{j}} \omega(b) \omega(u) \\
& \times\left[\frac{\partial}{\partial \alpha} B_{X}^{\alpha}\left(\boldsymbol{\Psi}_{j, u}, \boldsymbol{\Psi}_{j, u}\right)\right]\left(\alpha_{0}\right)\left[B_{X}^{\alpha_{0}}\left(\boldsymbol{\Psi}_{j, u}, \boldsymbol{\Psi}_{j, u}\right)\right]^{-1} \\
& \times\left[\frac{\partial}{\partial \alpha} B_{X}^{\alpha}\left(\boldsymbol{\Psi}_{j, b}, \mathbf{\Psi}_{j, b}\right)\right]\left(\alpha_{0}\right)\left[B_{X}^{\alpha_{0}}\left(\boldsymbol{\Psi}_{j, b}, \boldsymbol{\Psi}_{j, b}\right)\right]^{-1} \\
& \times \prod_{l=1}^{k-p} \mathscr{T}_{X}\left(\frac{\boldsymbol{\Psi}_{j, u}}{\left[B_{X}^{\alpha_{0}}\left(\mathbf{\Psi}_{j, u}, \mathbf{\Psi}_{j, u}\right)\right]^{1 / 2}}\right)\left(x_{l}\right) \\
& \times \prod_{l=k-p+1}^{2 k-2 p} \mathscr{T}_{X}\left(\frac{\boldsymbol{\Psi}_{j, b}}{\left[B_{X}^{\alpha_{0}}\left(\boldsymbol{\Psi}_{j, b}, \mathbf{\Psi}_{j, b}\right)\right]^{1 / 2}}\right)\left(x_{l}\right) \\
& \times\left[\prod_{l=1}^{p} \int_{D^{p} \times D^{p}} \frac{\boldsymbol{\Psi}_{j, u}\left(y_{l}\right)}{\left[B_{X}^{\alpha_{0}}\left(\boldsymbol{\Psi}_{j, u}, \boldsymbol{\Psi}_{j, u}\right)\right]^{1 / 2}}\right. \\
& \left.\times \frac{\boldsymbol{\Psi}_{j, b}\left(z_{l}\right)}{\left[B_{X}^{\alpha_{0}}\left(\mathbf{\Psi}_{j, b}, \mathbf{\Psi}_{j, b}\right)\right]^{1 / 2}} B_{\tilde{X}}\left(y_{l}, z_{l}\right) d y_{l} d z_{l}\right] d u d b \\
& =\kappa^{2}(j) \int_{L_{j} \times L_{j}} \omega(b) \omega(u) \\
& \times\left[\frac{\partial}{\partial \alpha} B_{X}^{\alpha}\left(\boldsymbol{\Psi}_{j, u}, \boldsymbol{\Psi}_{j, u}\right)\right]\left(\alpha_{0}\right)\left[B_{X}^{\alpha_{0}}\left(\boldsymbol{\Psi}_{j, u}, \boldsymbol{\Psi}_{j, u}\right)\right]^{-1} \\
& \times\left[\frac{\partial}{\partial \alpha} B_{X}^{\alpha}\left(\boldsymbol{\Psi}_{j, b}, \boldsymbol{\Psi}_{j, b}\right)\right]\left(\alpha_{0}\right)\left[B_{X}^{\alpha_{0}}\left(\boldsymbol{\Psi}_{j, b}, \boldsymbol{\Psi}_{j, b}\right)\right]^{-1} \\
& \times \prod_{l=1}^{k-p} \mathscr{T}_{X}\left(\frac{\boldsymbol{\Psi}_{j, u}}{\left[B_{X}^{\alpha_{0}}\left(\mathbf{\Psi}_{j, u}, \mathbf{\Psi}_{j, u}\right)\right]^{1 / 2}}\right)\left(x_{l}\right) \\
& \times \prod_{l=k-p+1}^{2 k-2 p} \mathscr{T}_{X}\left(\frac{\boldsymbol{\Psi}_{j, b}}{\left[B_{X}^{\alpha_{0}}\left(\boldsymbol{\Psi}_{j, b}, \mathbf{\Psi}_{j, b}\right)\right]^{1 / 2}}\right)\left(x_{l}\right) \\
& \times \frac{\left[B_{X}^{\alpha_{0}}\left(\mathbf{\Psi}_{j, b}, \boldsymbol{\Psi}_{j, u}\right)\right]^{p}}{\left[B_{X}^{\alpha_{0}}\left(\boldsymbol{\Psi}_{j, b}, \boldsymbol{\Psi}_{j, b}\right) B_{X}^{\alpha_{0}}\left(\boldsymbol{\Psi}_{j, u}, \boldsymbol{\Psi}_{j, u}\right)\right]^{p / 2}} d b d u .
\end{aligned}
$$

Its norm in the space $H^{\otimes 2 k-2 p}$ is then computed as follows:

$$
\begin{aligned}
\| \tilde{f}_{j}^{\alpha_{0}} & \otimes_{p} \tilde{f}_{j}^{\alpha_{0}} \|_{H^{\otimes 2 k-2 p}}=\int_{D^{2 k-2 p}} \kappa^{4}(j) \int_{L_{j} \times L_{j}} \int_{L_{j} \times L_{j}} \omega\left(b_{1}\right) \omega\left(u_{1}\right) \omega\left(b_{2}\right) \omega\left(u_{2}\right) \\
& \times\left[\frac{\partial}{\partial \alpha} B_{X}^{\alpha}\left(\boldsymbol{\Psi}_{j, b_{1}}, \mathbf{\Psi}_{j, b_{1}}\right)\right]\left(\alpha_{0}\right)\left[B_{X}^{\alpha}\left(\boldsymbol{\Psi}_{j, b_{1}}, \boldsymbol{\Psi}_{j, b_{1}}\right)\right]^{-1} \\
& \times\left[\frac{\partial}{\partial \alpha} B_{X}^{\alpha}\left(\boldsymbol{\Psi}_{j, u_{1}}, \boldsymbol{\Psi}_{j, u_{1}}\right)\right]\left(\alpha_{0}\right)\left[B_{X}^{\alpha_{0}}\left(\mathbf{\Psi}_{j, u_{1}}, \mathbf{\Psi}_{j, u_{1}}\right)\right]^{-1} \\
& \times\left[\frac{\partial}{\partial \alpha} B_{X}^{\alpha}\left(\mathbf{\Psi}_{j, b_{2}}, \boldsymbol{\Psi}_{j, b_{2}}\right)\right]\left(\alpha_{0}\right)\left[B_{X}^{\alpha}\left(\mathbf{\Psi}_{j, b_{2}}, \mathbf{\Psi}_{j, b_{2}}\right)\right]^{-1}
\end{aligned}
$$




$$
\begin{aligned}
& \times\left[\frac{\partial}{\partial \alpha} B_{X}^{\alpha}\left(\boldsymbol{\Psi}_{j, u_{2}}, \boldsymbol{\Psi}_{j, u_{2}}\right)\right]\left(\alpha_{0}\right)\left[B_{X}^{\alpha_{0}}\left(\boldsymbol{\Psi}_{j, u_{2}}, \boldsymbol{\Psi}_{j, u_{2}}\right)\right]^{-1} \\
& \times \frac{\left[B_{X}^{\alpha_{0}}\left(\boldsymbol{\Psi}_{j, b_{1}}, \boldsymbol{\Psi}_{j, u_{1}}\right)\right]^{p}}{\left[B_{X}^{\alpha_{0}}\left(\boldsymbol{\Psi}_{j, b_{1}}, \boldsymbol{\Psi}_{j, b_{1}}\right) B_{X}^{\alpha_{0}}\left(\boldsymbol{\Psi}_{j, u_{1}}, \boldsymbol{\Psi}_{j, u_{1}}\right)\right]^{p / 2}} \\
& \times \frac{\left[B_{X}^{\alpha_{0}}\left(\boldsymbol{\Psi}_{j, b_{2}}, \boldsymbol{\Psi}_{j, u_{2}}\right)\right]^{p}}{\left[B_{X}^{\alpha_{0}}\left(\boldsymbol{\Psi}_{j, b_{2}}, \boldsymbol{\Psi}_{j, b_{2}}\right) B_{X}^{\alpha_{0}}\left(\boldsymbol{\Psi}_{j, u_{2}}, \boldsymbol{\Psi}_{j, u_{2}}\right)\right]^{p / 2}} \\
& \times \prod_{l=1}^{k-p} \frac{\boldsymbol{\Psi}_{j, u_{1}}\left(x_{l}\right)}{\left[B_{X}^{\alpha_{0}}\left(\mathbf{\Psi}_{j, u_{1}}, \mathbf{\Psi}_{j, u_{1}}\right)\right]^{1 / 2}} \prod_{l=k-p+1}^{2 k-2 p} \frac{\boldsymbol{\Psi}_{j, b_{1}}\left(x_{l}\right)}{\left[B_{X}^{\alpha_{0}}\left(\mathbf{\Psi}_{j, b_{1}}, \mathbf{\Psi}_{j, b_{1}}\right)\right]^{1 / 2}} \\
& \times \prod_{l=1}^{k-p} \frac{\boldsymbol{\Psi}_{j, u_{2}}\left(y_{l}\right)}{\left[B_{X}^{\alpha_{0}}\left(\boldsymbol{\Psi}_{j, u_{2}}, \boldsymbol{\Psi}_{j, u_{2}}\right)\right]^{1 / 2}} \prod_{l=k-p+1}^{2 k-2 p} \frac{\boldsymbol{\Psi}_{j, b_{2}}\left(y_{l}\right)}{\left[B_{X}^{\alpha_{0}}\left(\boldsymbol{\Psi}_{j, b_{2}}, \mathbf{\Psi}_{j, b_{2}}\right)\right]^{1 / 2}} \\
& \times \prod_{l=1}^{2 k-2 p} B_{\tilde{X}}\left(x_{l}, y_{l}\right) d x_{l} d y_{l} d b_{1} d b_{2} d u_{1} d u_{2} \\
& =\kappa^{4}(j) \int_{L_{j} \times L_{j}} \int_{L_{j} \times L_{j}} \omega\left(b_{1}\right) \omega\left(b_{2}\right) \omega\left(u_{1}\right) \omega\left(u_{2}\right) \\
& \times\left[B_{X}^{\alpha_{0}}\left(\boldsymbol{\Psi}_{j, b_{1}}, \boldsymbol{\Psi}_{j, b_{1}}\right) B_{X}^{\alpha_{0}}\left(\boldsymbol{\Psi}_{j, b_{2}}, \boldsymbol{\Psi}_{j, b_{2}}\right)\right]^{-1} \\
& \times\left[B_{X}^{\alpha_{0}}\left(\boldsymbol{\Psi}_{j, u_{1}}, \boldsymbol{\Psi}_{j, u_{1}}\right) B_{X}^{\alpha_{0}}\left(\boldsymbol{\Psi}_{j, u_{2}}, \boldsymbol{\Psi}_{j, u_{2}}\right)\right]^{-1} \\
& \times\left[\frac{\partial}{\partial \alpha} B_{X}^{\alpha}\left(\boldsymbol{\Psi}_{j, b_{1}}, \boldsymbol{\Psi}_{j, b_{1}}\right)\right]\left(\alpha_{0}\right)\left[\frac{\partial}{\partial \alpha} B_{X}^{\alpha}\left(\boldsymbol{\Psi}_{j, b_{2}}, \boldsymbol{\Psi}_{j, b_{2}}\right)\right]\left(\alpha_{0}\right) \\
& \times\left[\frac{\partial}{\partial \alpha} B_{X}^{\alpha}\left(\boldsymbol{\Psi}_{j, u_{1}}, \boldsymbol{\Psi}_{j, u_{1}}\right)\right]\left(\alpha_{0}\right)\left[\frac{\partial}{\partial \alpha} B_{X}^{\alpha}\left(\boldsymbol{\Psi}_{j, u_{2}}, \boldsymbol{\Psi}_{j, u_{2}}\right)\right]\left(\alpha_{0}\right) \\
& \times \frac{\left[B_{X}^{\alpha_{0}}\left(\boldsymbol{\Psi}_{j, b_{1}}, \boldsymbol{\Psi}_{j, u_{1}}\right)\right]^{p}}{\left[B_{X}^{\alpha_{0}}\left(\boldsymbol{\Psi}_{j, b_{1}}, \boldsymbol{\Psi}_{j, b_{1}}\right) B_{X}^{\alpha_{0}}\left(\boldsymbol{\Psi}_{j, u_{1}}, \boldsymbol{\Psi}_{j, u_{1}}\right)\right]^{p / 2}} \\
& \times \frac{\left[B_{X}^{\alpha_{0}}\left(\boldsymbol{\Psi}_{j, b_{2}}, \boldsymbol{\Psi}_{j, u_{2}}\right)\right]^{p}}{\left[B_{X}^{\alpha_{0}}\left(\boldsymbol{\Psi}_{j, b_{2}}, \boldsymbol{\Psi}_{j, b_{2}}\right) B_{X}^{\alpha_{0}}\left(\mathbf{\Psi}_{j, u_{2}}, \mathbf{\Psi}_{j, u_{2}}\right)\right]^{p / 2}} \\
& \times\left[\frac{\left[B_{X}^{\alpha_{0}}\left(\boldsymbol{\Psi}_{j, b_{1}}, \boldsymbol{\Psi}_{j, b_{2}}\right)\right]^{k-p}}{\left[B_{X}^{\alpha_{0}}\left(\boldsymbol{\Psi}_{j, b_{1}}, \boldsymbol{\Psi}_{j, b_{1}}\right) B_{X}^{\alpha_{0}}\left(\boldsymbol{\Psi}_{j, b_{2}}, \boldsymbol{\Psi}_{j, b_{2}}\right)\right]^{(k-p) / 2}}\right] \\
& \times\left[\frac{\left[B_{X}^{\alpha_{0}}\left(\mathbf{\Psi}_{j, u_{1}}, \boldsymbol{\Psi}_{j, u_{2}}\right)\right]^{k-p}}{\left[B_{X}^{\alpha_{0}}\left(\mathbf{\Psi}_{j, u_{1}}, \mathbf{\Psi}_{j, u_{1}}\right) B_{X}^{\left(\alpha_{0}\right.}\left(\boldsymbol{\Psi}_{j, u_{2}}, \boldsymbol{\Psi}_{j, u_{2}}\right)\right]^{(k-p) / 2}}\right] d b_{1} d b_{2} d u_{1} d u_{2} .
\end{aligned}
$$

Considering the case $k=2$ and $p=1$, for $j$ sufficiently large, from Lemmas 3.1, the following approximation is considered:

$$
\begin{aligned}
& \left\|\tilde{f}_{j}^{\alpha_{0}} \otimes_{p} \tilde{f}_{j}^{\alpha_{0}}\right\|_{H^{\otimes 2 k-2 p}} \\
& \sim \frac{16\left[\ln \frac{1}{2}\right]^{4}}{\left[\widetilde{L}_{d}\left(\alpha_{0}\right]^{4}\right.} \int_{\left[0,2^{j}\right]^{4 d}} \omega\left(b_{1}\right) \omega\left(b_{2}\right) \omega\left(u_{1}\right) \omega\left(u_{2}\right) C_{\Psi}\left(\alpha_{0},\left\|b_{1}-u_{1}\right\|, \infty\right) \\
& \times C_{\boldsymbol{\Psi}}\left(\alpha_{0},\left\|b_{2}-u_{2}\right\|, \infty\right) C_{\boldsymbol{\Psi}}\left(\alpha_{0},\left\|b_{1}-b_{2}\right\|, \infty\right) \\
& \times C_{\Psi}\left(\alpha_{0},\left\|u_{1}-u_{2}\right\|, \infty\right) d b_{1} d b_{2} d u_{1} d u_{2} \\
& =K \int_{\left[-2^{j}, 2^{j}\right]^{d} \times\left[-2^{j}, 2^{j}\right]^{d} \times\left[-2^{j+1}, 2^{j+1}\right]^{d}}\left[\int_{D_{b_{1}}(h-\tau, z, h, j)} \omega\left(b_{1}\right) \omega\left(b_{1}-z\right)\right.
\end{aligned}
$$




$$
\begin{aligned}
& \left.\quad \times \omega\left(b_{1}-h\right) \omega\left(b_{1}+\tau-h\right) d b_{1}\right] \\
& \times C_{\Psi}\left(\alpha_{0},\|z\|, \infty\right) C_{\boldsymbol{\Psi}}\left(\alpha_{0},\|\tau\|, \infty\right) C_{\boldsymbol{\Psi}}\left(\alpha_{0},\|h-z\|, \infty\right) \\
& \times C_{\boldsymbol{\Psi}}\left(\alpha_{0},\|h-\tau\|, \infty\right) d h d \tau d z,
\end{aligned}
$$

where

$$
\begin{gathered}
K=\frac{16\left[\ln \frac{1}{2}\right]^{4}}{\left[\widetilde{L}_{d}\left(\alpha_{0}\right]^{4}\right.} \\
D_{b_{1}}(h-\tau, z, h, j)=\prod_{i=1}^{d}\left[h_{i}-\tau_{i}, h_{i}-\tau_{i}+2^{j}\right] \cap \prod_{i=1}^{d}\left[z_{i}, z_{i}+2^{j}\right] \cap \prod_{i=1}^{d}\left[h_{i}, h_{i}+2^{j}\right] .
\end{gathered}
$$

Denoting by

$$
I_{\omega}(z, \tau, h, j)=\int_{D_{b_{1}}(h-\tau, z, h, j)} \omega\left(b_{1}\right) \omega\left(b_{1}-z\right) \omega\left(b_{1}-h\right) \omega\left(b_{1}+\tau-h\right) d b_{1},
$$

equation (5.20) can be rewritten, and upper bounded, from Lemmas 3.1, as follows:

$$
\begin{aligned}
& \left\|\tilde{f}_{j}^{\alpha_{0}} \otimes_{p} \tilde{f}_{j}^{\alpha_{0}}\right\|_{H^{\otimes 2 k-2 p}} \sim K \int_{\left[-2^{j+1}, 2^{j+1}\right]^{d} \times\left[-2^{j}, 2^{j}\right]^{d} \times\left[-2^{j}, 2^{j}\right]^{d}} C_{\mathbf{\Psi}}\left(\alpha_{0},\|z\|, \infty\right) \\
& \times C_{\boldsymbol{\Psi}}\left(\alpha_{0},\|\tau\|, \infty\right) C_{\boldsymbol{\Psi}}\left(\alpha_{0},\|h-z\|, \infty\right) C_{\boldsymbol{\Psi}}\left(\alpha_{0},\|h-\tau\|, \infty\right) \\
& \times I_{\omega}(z, \tau, h, j) d \tau d z d h \\
& \leqslant K \int_{\left[-2^{j+1}, 2^{j+1}\right]^{d}}\left[\int_{\left[-2^{j}, 2^{j}\right]^{d} \backslash B_{R}(0) \times\left[-2^{j}, 2^{j}\right]^{d} \backslash B_{R}(0)} R^{4 \alpha_{0}-6 d}\right. \\
& \left.\times C_{\Psi}\left(\alpha_{0},\|h-z\|, \infty\right) C_{\Psi}\left(\alpha_{0},\|h-\tau\|, \infty\right) I_{\omega}(z, \tau, h, j) d \tau d z\right] d h \\
& +K \int_{\left[-2^{j+1}, 2^{j+1}\right]^{d}}\left[\int_{\left[-2^{j}, 2^{j}\right]^{d} \backslash B_{R}(0) \times B_{R}(0)} R^{2 \alpha_{0}-3 d}\left[\max _{x \in B_{R}(0)} \xi_{v}(x)\right]\right. \\
& \left.\times C_{\Psi}\left(\alpha_{0},\|h-z\|, \infty\right) C_{\Psi}\left(\alpha_{0},\|h-\tau\|, \infty\right) I_{\omega}(z, \tau, h, j) d \tau d z\right] d h \\
& +K \int_{\left[-2^{j+1}, 2^{j+1}\right]^{d}}\left[\int_{B_{R}(0) \times\left[-2^{j}, 2^{j}\right]^{d} \backslash B_{R}(0)} R^{2 \alpha_{0}-3 d}\left[\max _{x \in B_{R}(0)} \xi_{v}(x)\right]\right. \\
& \left.\times C_{\Psi}\left(\alpha_{0},\|h-z\|, \infty\right) C_{\Psi}\left(\alpha_{0},\|h-\tau\|, \infty\right) I_{\omega}(z, \tau, h, j) d \tau d z\right] d h \\
& +K \int_{\left[-2^{j+1}, 2^{j+1}\right]^{d}}\left[\int_{B_{R}(0) \times B_{R}(0)}\left[\max _{x \in B_{R}(0)} \xi_{v}(x)\right]^{2}\right. \\
& \left.\times C_{\boldsymbol{\Psi}}\left(\alpha_{0},\|h-z\|, \infty\right) C_{\boldsymbol{\Psi}}\left(\alpha_{0},\|h-\tau\|, \infty\right) I_{\omega}(z, \tau, h, j) d \tau d z\right] d h \\
& =K \int_{\left[-2^{j+1}-\frac{R}{\sqrt{d}}, 2^{j+1}+\frac{R}{\sqrt{d}}\right]^{d} \times\left[-2^{j+1}-\frac{R}{\sqrt{d}}, 2^{j+1}+\frac{R}{\sqrt{d}}\right]^{d}} \int_{D_{h}(x, y, j)} R^{4 \alpha_{0}-6 d}
\end{aligned}
$$




$$
\begin{aligned}
& \times C_{\boldsymbol{\Psi}}\left(\alpha_{0},\|x\|, \infty\right) C_{\boldsymbol{\Psi}}\left(\alpha_{0},\|y\|, \infty\right) I_{\omega}(h-x, h-y, h, j) d h d x d y \\
&+K \int_{D_{y}(j, R, d) \times\left[-2^{j+1}-\frac{R}{\sqrt{d}}, 2^{j+1}+\frac{R}{\sqrt{d}}\right]^{d}} R^{2 \alpha_{0}-3 d}\left[\max _{x \in B_{R}(0)} \xi_{v}(x)\right] C_{\boldsymbol{\Psi}}\left(\alpha_{0},\|x\|, \infty\right) C_{\boldsymbol{\Psi}}\left(\alpha_{0},\|y\|, \infty\right) \\
& \times \int_{D_{h}(x, y, j)} \times I_{\omega}(h-x, h-y, h, j) d h d x d y \\
&+K \int_{\left[-2^{j+1}-\frac{R}{\sqrt{d}}, 2^{j+1}+\frac{R}{\sqrt{d}}\right]^{d} \times D_{x}(j, R, d)} R^{2 \alpha_{0}-3 d}\left[\max _{x \in B_{R}(0)} \xi_{v}(x)\right] \\
& \times \int_{D_{h}(x, y, j)} C_{\boldsymbol{\Psi}}\left(\alpha_{0},\|x\|, \infty\right) C_{\boldsymbol{\Psi}}\left(\alpha_{0},\|y\|, \infty\right) \\
& \times I_{\omega}(h-x, h-y, h, j) d h d x d y \\
&+K \int_{D_{y}(j, R, d) \times D_{x}(j, R, d)}\left[\max _{x \in B_{R}(0)} \xi_{v}(x)\right]^{2} \\
& \times \int_{D_{h}(x, y, j)} C_{\boldsymbol{\Psi}}\left(\alpha_{0},\|x\|, \infty\right) C_{\boldsymbol{\Psi}}\left(\alpha_{0},\|y\|, \infty\right) \\
& \times I_{\omega}(h-x, h-y, h, j) d h d x d y \\
&=I_{1}(j)+I_{2}(j)+I_{3}(j)+I_{4}(j),
\end{aligned}
$$

where $D_{h}(x, y, j)$ and $D_{z}(j, R, d), z=x, y$, are given as in equation (3.2).

Applying again Lemma 3.1 to equation (5.22), in a similar way to equation (5.21), each one of the integrals $I_{i}(j), i=1,2,3,4$, can be decomposed into four integrals $I_{l, m}(j) ; l=1, \ldots, 4 ; m=1, \ldots, 4$, where similar upper bounds in terms of $R$ and $\left[\max _{x \in B_{R}(0)} \xi_{v}(x)\right]$ can be considered. Specifically, we obtain

$$
\left\|\tilde{f}_{j}^{\alpha_{0}} \otimes_{p} \tilde{f}_{j}^{\alpha_{0}}\right\|_{H \otimes 2 k-2 p} \leqslant \sum_{l=1}^{4} \sum_{m=1}^{4} f_{l, m}(R) g_{l, m}\left(\xi_{v}\right) I_{l, m}(j),
$$

where

$$
\begin{array}{r}
I_{l, m}(j)=\int_{D_{l, m}(j) \times D_{h}(x, y, j) \times D_{b_{1}}(h-x, y, h, j)} \omega\left(b_{1}\right) \omega\left(b_{1}-y\right) \\
\omega\left(b_{1}-h\right) \omega\left(b_{1}-(h-x)\right) d b_{1} d h d x d y
\end{array}
$$

with $D_{l, m}(j), l, m=1, \ldots, 4$, and $D_{b_{1}}(h-x, y, h, j)$ being defined as in equation (3.2). Under condition B.2, $I_{l, m}(j) \rightarrow 0$, as $j \rightarrow \infty$, for $l, m=$ $1, \ldots, 4$, and the Gaussian asymptotic distribution holds for $k=2$. Also, under condition B.2, from Cauchy-Schwarz inequality applied to equation (5.19), after considering Lemma 3.1, we obtain, for $k>2$, as $j \rightarrow \infty$, in a similar way to the case $k=2$, and $p=1$, that

$$
\begin{aligned}
& \left\|\tilde{f}_{j}^{\alpha_{0}} \otimes_{p} \tilde{f}_{j}^{\alpha_{0}}\right\|_{H \otimes 2 k-2 p} \\
& \quad \sim K_{1} \int_{L_{j} \times L_{j}} \int_{L_{j} \times L_{j}} \omega\left(b_{1}\right) \omega\left(b_{2}\right) \omega\left(u_{1}\right) \omega\left(u_{2}\right) \frac{\left[C_{\boldsymbol{\Psi}}\left(\alpha_{0}, b_{1}, u_{1}, \infty\right)\right]^{p}}{\left[\widetilde{L_{d}}\left(\alpha_{0}\right)\right]^{p}}
\end{aligned}
$$




$$
\begin{aligned}
& \times \frac{\left[C_{\boldsymbol{\Psi}}\left(\alpha_{0}, b_{2}, u_{2}, \infty\right)\right]^{p}}{\left[\widetilde{L_{d}}\left(\alpha_{0}\right)\right]^{p}} \frac{\left[C_{\boldsymbol{\Psi}}\left(\alpha_{0}, b_{1}, b_{2}, \infty\right)\right]^{k-p}}{\left[\widetilde{L_{d}}\left(\alpha_{0}\right)\right]^{k-p}} \\
& \times \frac{\left[C_{\Psi}\left(\alpha_{0}, u_{1}, u_{2}, \infty\right)\right]^{k-p}}{\left[\widetilde{L_{d}}\left(\alpha_{0}\right)\right]^{k-p}} d b_{1} d b_{2} d u_{1} d u_{2} \\
& \leqslant K_{1}\left[\int_{L_{j} \times L_{j}} \int_{L_{j} \times L_{j}} \omega\left(b_{1}\right) \omega\left(b_{2}\right) \omega\left(u_{1}\right) \omega\left(u_{2}\right) d b_{1} d b_{2} d u_{1} d u_{2}\right]^{1 / 2} \\
& \times\left[\int_{L_{j} \times L_{j}} \int_{L_{j} \times L_{j}} \omega\left(b_{1}\right) \omega\left(b_{2}\right) \omega\left(u_{1}\right) \omega\left(u_{2}\right) \frac{\left[C_{\boldsymbol{\Psi}}\left(\alpha_{0}, b_{1}, u_{1}, \infty\right)\right]^{2 p}}{\left[\widetilde{L_{d}}\left(\alpha_{0}\right)\right]^{2 p}}\right. \\
& \times \frac{\left[C_{\boldsymbol{\Psi}}\left(\alpha_{0}, b_{2}, u_{2}, \infty\right)\right]^{2 p}}{\left[\widetilde{L_{d}}\left(\alpha_{0}\right)\right]^{2 p}} \frac{\left[C_{\boldsymbol{\Psi}}\left(\alpha_{0}, b_{1}, b_{2}, \infty\right)\right]^{2(k-p)}}{\left[\widetilde{L_{d}}\left(\alpha_{0}\right)\right]^{2(k-p)}} \\
& \left.\times \frac{\left[C_{\Psi}\left(\alpha_{0}, u_{1}, u_{2}, \infty\right)\right]^{2(k-p)}}{\left[\widetilde{L_{d}}\left(\alpha_{0}\right)\right]^{2(k-p)}} d b_{1} d b_{2} d u_{1} d u_{2}\right]^{1 / 2} \\
& \leqslant K_{1}\left[\int_{L_{j}} \omega(b) d b\right]^{2}\left[\int_{L_{j} \times L_{j}} \int_{L_{j} \times L_{j}} \omega\left(b_{1}\right) \omega\left(b_{2}\right) \omega\left(u_{1}\right) \omega\left(u_{2}\right)\right. \\
& \times \frac{\left[C_{\boldsymbol{\Psi}}\left(\alpha_{0}, b_{1}, u_{1}, \infty\right)\right]^{2}}{\left[\widetilde{L_{d}}\left(\alpha_{0}\right)\right]^{2}} \frac{\left[C_{\boldsymbol{\Psi}}\left(\alpha_{0}, b_{2}, u_{2}, \infty\right)\right]^{2}}{\left[\widetilde{L_{d}}\left(\alpha_{0}\right)\right]^{2}} \\
& \left.\times \frac{\left[C_{\boldsymbol{\Psi}}\left(\alpha_{0}, b_{1}, b_{2}, \infty\right)\right]^{2}}{\left[\widetilde{L_{d}}\left(\alpha_{0}\right)\right]^{2}} \frac{\left[C_{\boldsymbol{\Psi}}\left(\alpha_{0}, u_{1}, u_{2}, \infty\right)\right]^{2}}{\left[\widetilde{L_{d}}\left(\alpha_{0}\right)\right]^{2}} d b_{1} d b_{2} d u_{1} d u_{2}\right]^{1 / 2} \\
& =K_{2} \int_{\left[-2^{j}, 2^{j}\right]^{d} \times\left[-2^{j}, 2^{j}\right]^{d} \times\left[-2^{j+1}, 2^{j+1}\right]^{d}} \\
& \times\left[\int_{D_{b_{1}}(h-\tau, z, h, j)} \omega\left(b_{1}\right) \omega\left(b_{1}-z\right) \omega\left(b_{1}-h\right) \omega\left(b_{1}+\tau-h\right) d b_{1}\right] \\
& \times\left[C_{\boldsymbol{\Psi}}\left(\alpha_{0},\|z\|, \infty\right) C_{\boldsymbol{\Psi}}\left(\alpha_{0},\|\tau\|, \infty\right) C_{\boldsymbol{\Psi}}\left(\alpha_{0},\|h-z\|, \infty\right)\right. \\
& \left.\times C_{\Psi}\left(\alpha_{0},\|h-\tau\|, \infty\right)\right]^{2} d h d \tau d z \\
& \leqslant K_{2} \sum_{l=1}^{4} \sum_{m=1}^{4} \widetilde{f_{l, m}}(R) \widetilde{g_{l, m}}\left(\xi_{v}\right) I_{l, m}(j) \longrightarrow 0
\end{aligned}
$$

where $K_{1}=16[\ln (1 / 2)]^{4}$ and $K_{2}=K_{1}\left[\int_{L_{j}} \omega(b) d b\right]^{2}\left[\widetilde{L}_{d}\left(\alpha_{0}\right)\right]^{-8}$ and $I_{l, m}(j)$, $l, m=1, \ldots, 4$, are defined as in equation (5.23).

Thus, from Lemma 4.1 , for every $k \geqslant 2$, the stochastic integral $I_{k}^{X}\left(\widetilde{f}_{j}^{\alpha_{0}}\right)$ converges in distribution to a Gaussian random variable with variance $k ! \sigma_{k}^{2}\left(\alpha_{0}\right)$, as $j \rightarrow \infty$. For a finite $n$, the convergence of

$$
S_{n}(j)=\sum_{k=1}^{n} \frac{C_{k}}{k !} I_{k}^{X}\left(\tilde{f}_{j}^{\alpha_{0}}\right),
$$

as $j \rightarrow \infty$, to a Gaussian distribution with variance

$$
\sum_{k=1}^{n} \frac{C_{k}^{2}}{k !} \sigma_{k}^{2}\left(\alpha_{0}\right),
$$


then follows from Corollary 4.1.

Convergence of the global Hermite expansion. The following lemma allows to establish the asymptotic normality of the minimum contrast estimator from the result previously derived on the convergence to the Gaussian distribution of the finite-dimensional Hermite sum.

As in the first part of the proof of Theorem 5.1, for each $k \geqslant n$, the sequence of kernels $\left\{\widetilde{f}_{j}^{\alpha_{0}}=\kappa(j) f_{j}^{\alpha_{0}}, j \geqslant 1\right\}$ is defined from the following identity:

$$
\begin{aligned}
f_{j}^{\alpha_{0}}\left(x_{1}, \ldots, x_{k}\right)=\int_{L_{j}} & \omega(b)\left[\frac{\partial}{\partial \alpha} B_{X}^{\alpha}\left(\mathbf{\Psi}_{j, b}, \mathbf{\Psi}_{j, b}\right)\right]\left(\alpha_{0}\right)\left[B_{X}^{\alpha_{0}}\left(\mathbf{\Psi}_{j, b}, \mathbf{\Psi}_{j, b}\right)\right]^{-1} \\
& \times \prod_{l=1}^{k} \mathscr{T}_{X}\left(\frac{\boldsymbol{\Psi}_{j, b}}{\left[B_{X}^{\alpha_{0}}\left(\mathbf{\Psi}_{j, b}, \mathbf{\Psi}_{j, b}\right)\right]^{1 / 2}}\right)\left(x_{l}\right) d b
\end{aligned}
$$

Lemma 5.1. Under the conditions assumed in Theorem 5.1, if for any $n \geqslant 1$, as $j \rightarrow \infty$,

$$
S_{n}(j)=\sum_{k=1}^{n} \frac{C_{k}}{k !} I_{k}^{X}\left(\widetilde{f}_{j}^{\alpha_{0}}\right)
$$

converges to a normal distribution with variance

$$
\sum_{k=1}^{n} \frac{C_{k}^{2}}{k !} \sigma_{k}^{2}\left(\alpha_{0}\right)
$$

then, the weak-convergence, as $n \rightarrow \infty$ and $j \rightarrow \infty$, of

$$
S_{n}(j)=\sum_{k=1}^{n} \frac{C_{k}}{k !} I_{k}^{X}\left(\tilde{f}_{j}^{\alpha_{0}}\right)
$$

to the normal distribution with variance

$$
\sigma^{2}=\sum_{k=1}^{\infty} \frac{C_{k}^{2}}{k !} \sigma_{k}^{2}\left(\alpha_{0}\right)
$$

also holds.

$\mathrm{P} r$ o o f. The proof follows from the convergence in probability to zero, when $n \rightarrow \infty$, of the tail

$$
\widetilde{S}_{n}(j)=\sum_{k=n}^{\infty} \frac{C_{k}}{k !} I_{k}^{X}\left(\widetilde{f}_{j}^{\alpha_{0}}\right),
$$

uniformly in $j$, under conditions of Theorem 5.1. Specifically, for $n \geqslant 2$, applying Cauchy-Schwarz inequality, for $j$ sufficiently large,

$$
\mathbf{E}\left(\widetilde{S}_{n}(j)\right)^{2}=\mathbf{E}\left(\sum_{k=n}^{\infty} \frac{C_{k}}{k !} I_{k}^{X}\left(\tilde{f}_{j}^{\alpha_{0}}\right)\right)^{2}
$$




$$
\begin{aligned}
& =\sum_{k=n}^{\infty} \frac{C_{k}^{2}}{k !} \kappa^{2}(j) \int_{L_{j} \times L_{j}} \omega(b)\left[\frac{\partial}{\partial \alpha} B_{X}^{\alpha}\left(\boldsymbol{\Psi}_{j, b}, \mathbf{\Psi}_{j, b}\right)\right]\left(\alpha_{0}\right)\left[B_{X}^{\alpha_{0}}\left(\mathbf{\Psi}_{j, b}, \boldsymbol{\Psi}_{j, b}\right)\right]^{-1} \\
& \times \omega(u)\left[\frac{\partial}{\partial \alpha} B_{X}^{\alpha}\left(\boldsymbol{\Psi}_{j, u}, \boldsymbol{\Psi}_{j, u}\right)\right]\left(\alpha_{0}\right)\left[B_{X}^{\alpha_{0}}\left(\boldsymbol{\Psi}_{j, u}, \boldsymbol{\Psi}_{j, u}\right)\right]^{-1} \\
& \times \frac{\left[B_{X}^{\alpha_{0}}\left(\boldsymbol{\Psi}_{j, b}, \boldsymbol{\Psi}_{j, u}\right)\right]^{k}}{\left[B_{X}^{\alpha_{0}}\left(\mathbf{\Psi}_{j, b}, \boldsymbol{\Psi}_{j, b}\right)\right]^{k / 2}\left[B_{X}^{\alpha_{0}}\left(\mathbf{\Psi}_{j, u}, \mathbf{\Psi}_{j, u}\right)\right]^{k / 2}} d u d b \\
& \leqslant \sigma_{2}^{2}\left(\alpha_{0}\right) \sum_{k=n}^{\infty} \frac{C_{k}^{2}}{k !}=F(n) \underset{n \rightarrow \infty}{\longrightarrow} 0 \text {, }
\end{aligned}
$$

since by Parseval identity $\sum_{k=1}^{\infty}\left(C_{k}^{2} / k !\right)<\infty$. The rest of the proof follows as in [19]. Specifically, we consider the following probabilities:

$$
\mathbf{P}\left\{\left|\widetilde{S}_{n}(j)\right|>\varepsilon\right\} \leqslant \frac{F(n)}{\varepsilon^{2}} \rightarrow 0, \quad n \rightarrow \infty .
$$

Moreover, for $S(j)=S_{n}(j)+\widetilde{S}_{n}(j)$,

$$
\begin{aligned}
\mathbf{P}\{S(j) \leqslant x\} & =\mathbf{P}\left\{S(j) \leqslant x,\left|\widetilde{S}_{n}(j)\right| \leqslant \varepsilon\right\}+\mathbf{P}\left\{S(j) \leqslant x,\left|\widetilde{S}_{n}(j)\right|>\varepsilon\right\} \\
& \leqslant \mathbf{P}\left\{S_{n}(j) \leqslant x+\varepsilon\right\}+\frac{F(n)}{\varepsilon^{2}} .
\end{aligned}
$$

Thus, for any $\varepsilon>0$, and $n \geqslant 1$

$$
\varlimsup_{j \rightarrow \infty} \mathbf{P}\{S(j) \leqslant x\} \leqslant \Phi_{n}(x+\varepsilon)+\frac{F(n)}{\varepsilon^{2}},
$$

where $\Phi_{n}$ is a cumulative distribution function of a Gaussian r.v. with zero mean and variance $\sigma_{n}^{2}(z)=\sum_{k=1}^{n}\left(C_{k}^{2} / k !\right) \sigma_{k}^{2}\left(\alpha_{0}\right)$.

On the other hand,

$$
\begin{aligned}
\mathbf{P}\left\{S_{n}(j) \leqslant x-\varepsilon\right\} \leqslant & \mathbf{P}\left\{S_{n}(j)+\varepsilon \leqslant x,\left|\widetilde{S}_{n}(j)\right| \leqslant \varepsilon\right\} \\
& +\mathbf{P}\left\{S_{n}(j)+\varepsilon \leqslant x,\left|\widetilde{S}_{n}(j)\right|>\varepsilon\right\} \\
\leqslant & \mathbf{P}\{S(j) \leqslant x\}+\frac{F(n)}{\varepsilon^{2}},
\end{aligned}
$$

that is, for any $\varepsilon>0$, and $n \geqslant 1$, as $j \rightarrow \infty$,

$$
\underline{\lim } \mathbf{P}\{S(j) \leqslant x\} \geqslant \Phi_{n}(x-\varepsilon)-\frac{F(n)}{\varepsilon^{2}} .
$$

If $n \rightarrow \infty$, we obtain from equations (5.27) and (5.28) that, for every $j \geqslant 1$,

$$
\Phi_{\infty}(x-\varepsilon) \leqslant \underline{\lim } \mathbf{P}\{S(j) \leqslant x\} \leqslant \varlimsup \lim \mathbf{P}\{S(j) \leqslant x\} \leqslant \Phi_{\infty}(x+\varepsilon),
$$


where $\Phi_{\infty}$ is a cumulative distribution function of a Gaussian r.v. with zero mean and the variance

$$
\sigma^{2}(z)=\sum_{k=1}^{\infty} \frac{C_{k}^{2}}{k !} \sigma_{k}^{2}\left(\alpha_{0}\right) .
$$

Thus, if $\varepsilon \rightarrow 0, \lim _{j \rightarrow \infty} \mathbf{P}\{S(j) \leqslant x\}=\Phi_{\infty}(x), x \in \mathbf{R}$. Lemma 5.1 is proved.

6. Final comments. The asymptotic normality of the wavelet-based minimum contrast estimator proposed in [36] is obtained from the application of the central limit result derived in [29]. The main step of the proof consists of the identification of the wavelet random field with an isonormal process (see Lemma 4.3). This step allows us to establish the convergence to the Gaussian distribution from the application of Lemma 4.1. Thus, we adopt an alternative methodological line to the method of moments and diagram formulae, based on the the convergence to zero of the norms of the self-contractions of the symmetric kernels involved in the construction of the sequence of multiple stochastic integrals under study. In our context, this sequence is formulated from the Hermite expansion of the integral weighted non-linear functional of the wavelet random field, defining the derivative of the contrast process. The increasing domain asymptotic approach in the wavelet domain is considered in the derivation of the mentioned limit results. This approach opens new research lines in the investigation of fixed-domain asymptotic properties of estimators in a parametric or semiparametric framework. The same idea can be applied to the nonparametric estimator defined in terms of the wavelet periodogram. The flexibility of the wavelet domain, due to the existence of a large variety of wavelet bases satisfying different regularity and moment conditions, allows the design of a vast class of contrast functions. In addition, the non-stationary Gaussian random field case can be treated in a suitable way.

The extension of the derived results to a non-Gaussian framework can be addressed, in the linear random field case, from the central limit results previously obtained for integral non-linear functionals and bilinear forms involving Appell polynomials (see [15], [38], where limit results for moving average sequences with long-range dependence are formulated, and [8], where an extension to the case of multidimensional indices of the functional analysis approach proposed by [7] is derived).

Acknowledgements. We would like to thank Prof. N. Leonenko for his helpful comments and suggestions that have considerably improved this paper. We also thank the referee for his/her suggestions. 


\section{REFERENCES}

1. Adler R. J. The Geometry of Random Fields. New York: John Wiley, 1981, 280 p.

2. Abry P. Transformées en ondelettes Analyses multirésolutions et signaux de pression en turbulance. - Doct. diss. Univ. Claude Bernard, France, Lyon, 1994.

3. Ahn V., Angulo J.M., Ruiz-Medina M.D. Possible long-range dependence in fractional random fields. - J. Statist. Plann. Inference, 1999, v. 80, p. 95-110.

4. Ahn V., Leonenko N. N., Sakhno L. M. On a class of minimum contrast estimators for fractional stochastic processes and fields. - J. Statist. Plann. Inference, 2004, v. 123, p. $161-185$.

5. Ahn V., Leonenko N. N., Sakhno L. M. Minimum contrast estimation of random processes based on information of second and third orders. - J. Statist. Plann. Inference, 2007, v. 137, p. 1302-1331.

6. Antoniadis A., Gijbels I. Detecting abrupt changes by wavelet methods. - J. Nonparamet. Stat., 2002, v. 14, № 1,2, p. 7-29.

7. Avram F., Brown L. A generalized Hölder inequality and a generalized Szego theorem. - Proc. Amer. Math. Soc., 1989, v. 107, № 3, p. 687-695.

8. Avram F., Leonenko N., Sakhno L. On a Szegö type limit theorem, the Hölder-YoungBrascamp-Lieb inequality and the asymptotic theory of integrals and quadratic forms of stationary fields. - ESAIM Probab. Stat., 2010, v. 14, p. 210-255.

9. Chan G., Wood A.T.A. Estimation of fractal dimension for a class of non-Gaussian stationary processes and fields. - Ann. Statist., 2004, v. 32, № 3, p. 1222-1260.

10. Corcuera J.M., Nualart D., Woerner J. H. C. Convergence of certain functionals of integral fractional processes. - J. Theoret. Probab., 2009, v. 22, № 4, p. 856-870.

11. Dahlhaus R., Künsch $H$. Edge effects and efficient parameter estimation for stationary random fields. - Biometrika, 1987, v. 74, № 4, p. 877-882.

12. Du J., Zhang H., Mandrekar V.S. Fixed-domain asymptotic properties of typed maximum likelihood estimators. - Ann. Statist., 2009, v. 37, №6A, p. 3330-3361.

13. Fay G., Moulines E., Roueff F., Taqqu M.S. Estimators of long-memory: Fourier versus wavelets. - J. Econometrics, 2009, v. 15, № 2, p. 159-177.

14. Gao J., Ahn V., Heyde C. Statistical estimation of nonstationary Gaussian processes with long-range dependence and intermittency. - Stochastic Process. Appl., 2002, v. 99, № 2, p. 295-321.

15. Giraitis L., Taqqu M.S. Central limit theorems for quadratic forms with time domain conditions. - Ann. Probab., 1998, v. 26, № 1, p. 377-398.

16. Guyon $X$. Parameter estimation for a stationary process on a d-dimensional lattice. Biometrika, 1982, v. 69, № 1, p. 95-105.

17. Guyon X. Random Fields on a Network: Modelling, Statistics and Applications. New York: Springer-Verlag, 1995, $225 \mathrm{p}$.

18. Heyde C. C. Quasi-likelihood and its Applications. A General Approach to Optimal Parameter Estimation. New York: Springer-Verlag, 1997, 235 p.

19. Ivanov A. V., Leonenko N. N., Ruiz-Medina M. D., Savich I. N. (2010). Limit theorems for weighted nonlinear transformations of Gaussian processes with singular spectra. Ann. Probab., 2013, v. 41, № 2, p. 1088-1114.

20. Leonenko N. N., Sakhno L.M. On the Whittle estimators for some classes of continuous-parameter random processes and fields. - Statist. Probab. Lett., 2003, v. 76 , № 8 , p. $781-795$.

21. Lim, Ch. Y., Stein M. L. Properties of spatial cross-periodograms using fixed-domain asymptotics. - J. Multivatiate Anal., 2008, v. 99, № 9, p. 1962-1984.

22. Ludeña C. Parametric estimation for Gaussian long-range dependent processes based on the log-periodogram. - Bernoulli, 2000, v. 6, № 4, p. 709-728.

23. Mallat S., Hwang W. L. Singularity detection and processing with wavelets. - IEEE Trans. Inform. Theory, 1992, v. 38, № 2, p. 617-643.

24. Nason G.P., Silverman B. W. The stationary wavelet transform and some statistical applications. - Lecture Notes in Statist., 1994, v. 103, p. 281-300.

25. Nason G.P., von Sachs R., Kroisandt G. Wavelet processes and adaptive estimation of evolutionary wavelet spectrum. - J. Royal Statist. Soc., Ser. B, 2000, v. 62, p. 271-292. 
26. Nourdin I., Nualart D. Central limit theorems for multiple Skorokhod integrals. - J. Theoret. Probab., 2010, v. 23, № 1, p. 39-64.

27. Nualart D. The Malliavin Calculus and Related Topics. New York: Springer-Verlag, 1995, $266 \mathrm{p}$.

28. Nualart D., Ortiz-Latorre $S$. Central limit theorems for multiple stochastic integrals and Malliavin calculus. - Stochastic Process. Appl., 2008, v. 118, № 4, p. 614-628.

29. Nualart D., Peccati G. Central limit theorems for sequences of multiple stochastic integrals. - Ann. Probab, 2005, v. 33, № 1, p. 91-115.

30. Peccati G., Tudor C.A. Gaussian limits for vector-valued multiple stochastic integrals. - Séminaire de Probabilités XXXVIII, Lecture Notes in Math., v. 1857, Spriner, Berlin, 2005, p. 247-262.

31. Peccati G., Taqqu M.S. Stable convergence of multiple Wiener-Itô integrals. - J. Theoret. Probab., 2008, v. 21, p. 527-570.

32. Peccati G., Taqqu M.S. Limit theorems for multiple stochastic integrals. - ALEA Lat. Amer. J. Probab. Math. Statist., 2008, v. 4, p. 393-413.

33. Peccati G., Taqqu M.S. Stable convergence of generalized $L^{2}$ stochastic integrals and the principle of conditioning. - Electron. J. Probab., 2007, v. 12, № 15, p. 447-480.

34. Roueff F., Taqqu M.S. Asymptotic normality of wavelet estimation of the memory parameter for linear processes. - J. Time Series Anal., 2009, v. 30, № 5, p. 534-558.

35. Ruiz-Medina M.D., Angulo J.M., Anh V.V. Fractional generalized random fields on bounded domains. - Stochastic Anal. Appl., 2003, v. 21, № 2, p. 465-492.

36. Ruiz-Medina M.D., Crujeiras R. M. Minimum contrast parameter estimation for fractal random fields based on the wavelet periodogram. - Comm. Statist. Theory Methods, 2011, v. 40, № 19-20, p. 3599-3613.

37. Stein M. L. Fixed-domain asymptotics for spatial periodograms. - J. Amer. Statist. Assoc., 1995, v. 432, p. 1277-1288.

38. Surgailis D. Long-range dependence and Appell rank. - Ann. Probab., 2000, v. 28, № 1, p. 478-497.

39. Taniguchi $M$. Minimum contrast estimation for spectral densities of stationary processes. - J. Roy. Statist. Soc., Ser. B, 1987, v. 49, № 3, p. 315-325.

40. Triebel H. Interpolation Theory, Function Spaces, Differential Operators. Amsterdam-New York: North-Holland Publishing Co., 1978, 528 p.

41. Vakhania N. N., Tarieladze V.I.kb Chobanyan S. A. Probability Distributions on Banach Spaces. Dordrecht: D. Reidel Publishing Co., 1987, 482 p.

Поступила в редакцию

14.III.2011

Исправленный вариант

14.XII.2011 